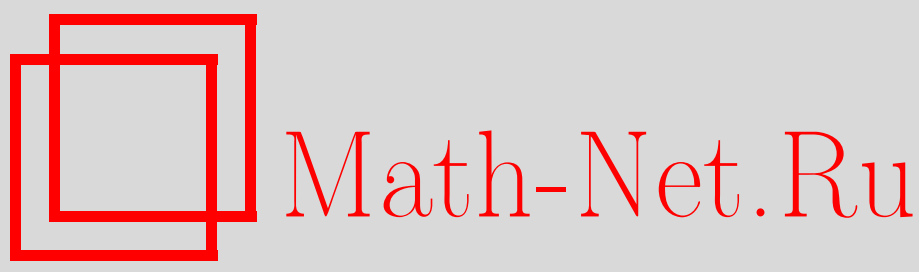

И. В. Артамкин, Канонические отображения пунктированных кривых с простейшими особенностями, Матем. сб., 2004, том 195, номер 5, 3-32

DOI: https://doi.org/10.4213/sm818

Использование Общероссийского математического портала Math-Net.Ru подразумевает, что вы прочитали и согласны с пользовательским соглашением

http://www . mathnet.ru/rus/agreement

Параметры загрузки:

IP: 54.166 .219 .16

26 апреля 2023 г., 15:15:11 
УДК 512.772

\author{
И.В. Артамкин
}

\title{
Канонические отображения пунктированных кривых с простейшими особенностями
}

В 1983 году Кнудсен доказал, что трижды каноническое отображение стабильной по Делиню-Мамфорду пунктированной кривой является вложением, а дважды каноническое не имеет базисных точек. Здесь обсуждается тот же вопрос для канонического отображения. Ответ можно сформулировать почти чисто топологически в терминах двойственного граффа, за исключением случая гиперэллиптических кривых.

Библиографиял: 7 названий.

\section{§1. Введение}

В этой работе рассматриваются особые кривые над алгебраически замкнутым полем $k$, имеющие простейшие двойные особые точки. Предположим, что такая кривая $X$ имеет неприводимые компоненты $X_{v}, v \in V$, и особые точки $p_{e}$,

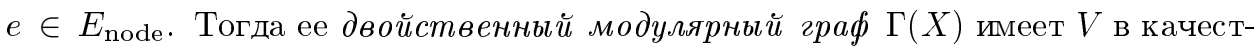

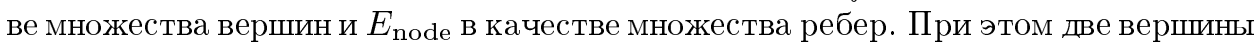
$v_{1}$ и $v_{2}$ соединяются ребром $e$ тогда и только тогда, когда ветви особой точки $p_{e}$ принадлежат компонентам $X_{v_{1}}$ и $X_{v_{2}}$. Таким образом, точки пересечения двух различных компонент соответствуют ребрам с двумя различными концами, в то время как точка самопересечения одной компоненты соответствует петле.

Мы будем рассматривать также пунктированные кривые, т.е. кривые, на которых отмечено некоторое конечное (возможно, пустое) множество неособых точек $p_{e}, e \in E_{\mathrm{par}}$. Отмеченной точке $p_{e}$ мы будем сопоставлять в модулярном графе $\Gamma(X)$ параболические ребра, т.е. ребра, имеющие только один конец - такую вершину графа $v$, что $p_{e} \in X_{v}$. Таким образом, дуальный модулярный граф̆ пунктированной кривой имеет множество $V$ в качестве множества вершин и множество $E=E_{\text {node }} \cup E_{\text {par }}-$ в качестве множества ребер, при этом мы будем рассматривать обычные и параболические ребра с единой точки зрения. Например, условие инцидентности вершины $v$ и ребра $e$ в любом случае записьвается одинаково: $p_{e} \in X_{v}$. Отметим еше, что пунктированную кривую можно также рассматривать и как некомпактную кривую, полученную из компактной выбрасыванием отмеченных точек $p_{e}, e \in E_{\text {par. }}$. Соответственно пунктированную кривую с пустым множеством отмеченных точек мы иногда будем называть компактной, а соответствующий ей модулярный граф - компактныцм модулярныцм графом.

Модулярный граф $\Gamma(X)$ является неориентированным графом; каждому его ребру (в том числе и петле) можно при желании приписать любую из двух возможных ориентаций. Обозначим множество ориентированных ребер графа $\Gamma(X)$ 
через $\vec{E}$; для ориентированного ребра $\vec{e}$ обозначим через $s(\vec{e})$ вершину, из которой выходит ребро $\vec{e}$, а через $t(\vec{e})-$ вершину, в которую входит ребро $\vec{e}$. Множество $S_{-}(v)=s^{-1}(v)$ называется звездой исходящих из вершины $v$ ребер; множество

$$
\overrightarrow{E_{1}}=\bigcup_{v \in V} S_{-}(v)
$$

представляет собой множество всех исходящих (из какой-либо вершины) ориентированных ребер графа $\Gamma(X)$, так что $s$ является отображением

$$
s: \overrightarrow{E_{1}} \rightarrow V .
$$

Отображения $s$ и $t$ сопряжены относительно инволюции смены ориентации

$$
i: \vec{E} \rightarrow \vec{E}
$$

т.e.

$$
t=s \circ i: i\left(\overrightarrow{E_{1}}\right) \rightarrow V .
$$

Для компактного графа, очевидно, $\overrightarrow{E_{1}}=\vec{E}$.

Нормализация $X^{\nu}$ кривой $X$ является несвязным объединением неособых криBux

$$
X^{\nu}=\coprod_{v \in V} X_{v}^{\nu}
$$

а отображение нормализации $\nu: X^{\nu} \rightarrow X$ представляет собой просто набор отображений нормализации компонент $\nu_{v}: X_{v}^{\nu} \rightarrow X_{v}, v \in V$. Имеется естественное взаимно однозначное соответствие между прообразами $\nu^{-1} p_{e}$ точек $p_{e}, e \in E$, лежащих на компоненте $X_{v}$, и звездой исходящих ребер $S_{-}(v)$, поэтому мы будем обозначать точку на $X_{v}^{\nu}$, соответствуюшую ориентированному ребру $\vec{e} \in S_{-}(v)$, через $P_{\vec{e}}$. Это сопоставление положено в основу формального определения модулярного графа, приведенного в начале следующего параграфа; отметим, что часто встречающееся определение, основанное на использовании флагов (см., например, [1]), отличается от нашего, использушего все ориентированные ребра.

Пучок дифференциальных форм $\Omega_{X}^{1}$ на $X$ локально свободен; ограничение его подъема $\nu^{*} \Omega_{X}^{1}$ на компоненту $X_{v}^{\nu}$ представляет собой пучок дифференциальных форм третьего рода на $X_{v}^{\nu}$, имеющих полюсы лишь в точках $P_{\vec{e}}, \vec{e} \in S_{-}(v)$ :

$$
\left.\left(\nu^{*} \Omega_{X}^{1}\right)\right|_{X_{v}^{\nu}}=\Omega_{X_{v}^{\nu}}^{1}\left(\sum_{\vec{e} \in S_{-}(v)} P_{\vec{e}}\right) .
$$

Таким образом, регулярный дифференциал на $X$ представляет собой набор дифференциалов третьего рода на каждой $X_{v}^{\nu}$, имеюших полюсы лишш в точках $P_{\vec{e}}$, $\vec{e} \in S_{-}(v)$.

Каждая особая точка $p_{e}, e \in E_{\text {node }}$, налагает одно условие на дифференциалы $\left\{\omega_{v}\right\}$ : если е соединяет вершины $v_{1}$ и $v_{2}$, то

$$
\operatorname{res}_{P_{\vec{e}}} \omega_{v_{1}}+\operatorname{res}_{P_{\overparen{e}}} \omega_{v_{2}}=0
$$

(Здесь и далее $\vec{e}$ и $\overleftarrow{e}$ обозначают две возможные ориентации на ребре $e$, так что $s(\vec{e})=t(\overleftarrow{e})=v_{1}$ и $s(\overleftarrow{e})=t(\vec{e})=v_{2}$.) Таким образом, набор вычетов в ветвях двойных особых точек является кососимметрическим, более того, его можно 
продолжить до кососимметрической функции на всем $\vec{E}$, сопоставляя - res $P_{\overleftarrow{e}} \omega_{v}$ ориентированному параболическому ребру $\vec{e} \notin \overrightarrow{E_{1}}$, направленному к вершине $v$. Основная теорема о вычетах для каждой компоненты $X_{v}^{\nu}$ дает тогда условие коцикла:

$$
\sum_{\vec{e} \in S_{-}(v)} \operatorname{res}_{P_{\vec{e}}} \omega_{v}=0
$$

Отображение, сопоставляюшее дифференциалу $\omega \in H^{0}\left(X, \Omega_{X}^{1}\right)$ набор значений его вычетов во всех точках $P_{\vec{e}}, \vec{e} \in \vec{E}$, являющееся аналогом отображения периодов для пунктированных особых кривых, будет подробно обсуждаться в следующем параграфе.

Нас будет интересовать $m$-каноническое отображение

$$
\varphi_{m}: X \rightarrow \mathbb{P}\left(H^{0}\left(X,\left(\Omega_{X}^{1}\right)^{\otimes m}\right)^{*}\right)
$$

В работе [2] доказано, что для стабильной в смысле Делиня-Мамфорда (см. [3]) кривой $X$ отображение $\varphi_{m}$ не имеет базисных точек при $m \geqslant 2$ и является вложением при $m \geqslant 3$. В следующей теореме дается условие того, что дважды каноническое отображение является вложением.

Теорема 1.1. Дважды каноническое отображение стабильной в смысле Делиня-Мамфорда кривой $X$ является влохсением, если:

1) модулярный граф $Г(X)$ не содержит одновалентной вершины $v$, для которой $g\left(X_{v}\right)=1$;

2) модулярный граф $\Gamma(X)$ не содержст трехвалентной вершины $v$ с петлей, для которой $g\left(X_{v}\right)=0$;

3) $X$ не является одной из следующих четырех компактных (т.е. не имеющих отмеченных точек) кривых рода 2:

а) неособая кривая рода 2,

b) әллиптическая кривая с двойной особой точкой,

c) рачиональная кривая с двумя двойными особыми точками,

d) пара неособых рачиональных кривых, трансверсально пересекающихся в трех различных точках.

На каждой неприводимой компоненте типа 1) и 2) и на каждой кривой типа 3) $\varphi_{2}$ является двулистным накрытием проективной прямой.

Поведение канонического отображения зависит от следуюшего топологического инварианта модулярного графа $\Gamma(X)$. Пусть $\Gamma$ - произвольньй модулярньй граф̆, не обязательно связный. Обозначим через $R(\Gamma)$ множество его компактных связных компонент (т.е. связных компонент, не содержащих параболических ребер).

ОПРЕДЕЛЕНИЕ 1.1. Толщиной ${ }^{1} \operatorname{th}(Г)$ модулярного графа Г называется наименьшее количество ребер графа $\Gamma$, которое необходимо удалить, чтобы увеличить число $|R(\Gamma)|$.

\footnotetext{
${ }^{1}$ В теории граффов принята другая терминология. Здесь мы следуем [5].
} 
Очевидно, $\operatorname{th}(\Gamma)$ не превосходит минимальной валентности вершин графа $\Gamma$, поскольку удаление всех исходящих из данной вершины ребер делает эту вершину новой компактной связной компонентой. Для связного некомпактного модулярного графа th $(\Gamma)$ не превосходит числа его параболических ребер $\left|E_{\text {par }}\right|$, поскольку в этом случае удаление всех параболических ребер увеличивает $|R(\Gamma)|$ с 0 до 1 . На рис. 1, 2 и 3 приведены примеры графов толшины соответственно 1,2 и 3.
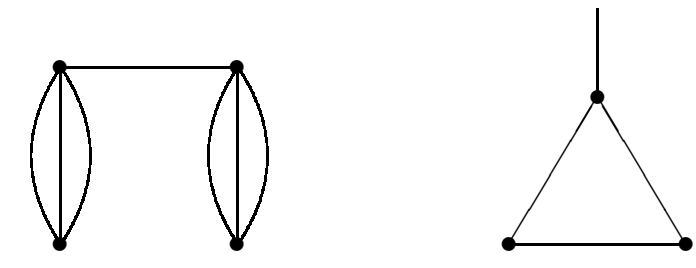

Рис. 1. Модулярные графы толщины 1
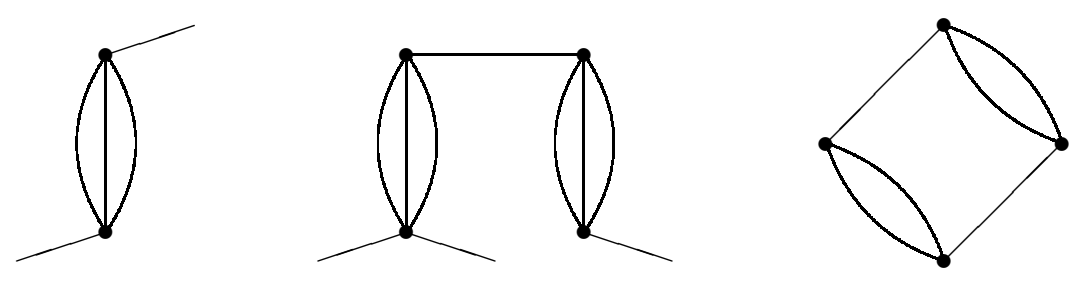

Рис. 2. Модулярные граффы толщины 2
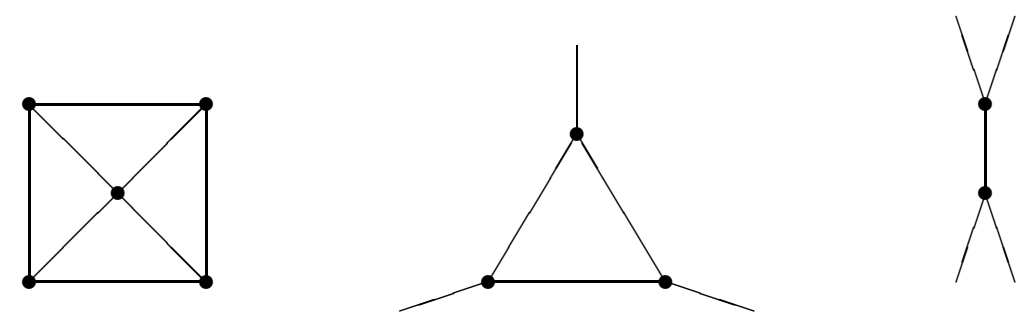

Рис. 3. Модулярные графы толщины 3

Размерность $g_{\gamma}(X)$ пространства регулярных дифференциальных форм на кривой $X$ может быть выражена через роды нормализаций неприводимых компонент $X$ и основные характеристики двойственного модулярного графа $\Gamma(X)$ :

$$
g_{\gamma}=\sum_{v \in V} g\left(X_{v}^{\nu}\right)+|E|-|V|+|R| .
$$

Для компактной (т.е. не имеющей отмеченных точек) кривой с двойными точками арифметический род совпадает с $g_{\gamma}$; добавление отмеченных точек, конечно, не меняет арифметический род, но может изменить $g_{\gamma}$. 
Оказывается, почти все кривые $\mathrm{c}$ th $(\Gamma(X)) \geqslant 3$ стабильны в смысле Делиня-Мамфорда.

ПРЕДЛОЖЕНИЕ 1.1. Если $\operatorname{th}(\Gamma(X)) \geqslant 3 u g_{\gamma}(X)>1$, то кривая $X$ стабильна в смысле Делиня-Мамфорда.

Отметим, что имеется всего три связные кривые $\mathrm{c} \operatorname{th}(\Gamma(X)) \geqslant 3$ и $g_{\gamma}(X) \leqslant 1$, причем все они компактны (т.е. не имеют отмеченных точек). Это проективная прямая $\left(g_{\gamma}=0\right)$, неособая эллиптическая кривая $\left(g_{\gamma}=1\right)$ и рациональная кривая с одной двойной точкой $\left(g_{\gamma}=1\right)$.

Основным результатом этой работы является следующая теорема.

ТЕОРемА 1.2. ${ }^{2}$ Каноническое отображение пунктированной особой кривой $X$ с простейшими двойными точками:

I. Не имеет базисных точек тогда и только тогда, когда $\operatorname{th}(\Gamma(X)) \geqslant 2$.

II. Является вложснием в $\mathbb{P}^{g_{\gamma}-1}$ тогда и только тогда, когда толщина графа $\operatorname{th}(\Gamma(X)) \geqslant 3, g_{\gamma}(X)>1$ и кривая $X$ не является обобщенной гиперәллиптической кривой (см. п. III настоящей теоремъ); образ канонического отображения является кривой степени

$$
d_{\gamma}=2\left(g_{\gamma}-|R|\right)-\left|E_{\mathrm{par}}\right|
$$

III. Является двойным накрытием рациональной кривой Веронезе степени $g_{\gamma}-1$ в $\mathbb{P}_{\gamma}-1$ для следующих двух типов кривых (которье миь будем называть обобщеннымм гиперэллиптическими, см. рис. 4):

а) неприводимая кривая $X$ с рачиональной, әллиптической или гиперәллиптической кривой $X^{\nu}$ с инволючией $i: X^{\nu} \rightarrow X^{\nu}$, переставляюшей ветви всех $n \geqslant \max \left(2-g\left(X^{\nu}\right), 0\right)$ особых точек: $i\left(P_{\overleftarrow{e}}\right)=P_{\vec{e}}$ для всех п ребер (которые являются петлями) гра$\oint a \Gamma(X)$

b) приводимая кривая $X=X_{1} \cup X_{2}$, имеющая две неособъе рациональные неприводимые компоненты $X_{1} u X_{2}$, попарно пересекающиеся в $n \geqslant 3$ двойных особъх точках таким образом, что имеется проективный изоморфизм $i: X_{1} \rightarrow X_{2}$, переставляющий ветви особъх точек: $i\left(P_{\overleftarrow{e}}\right)=P_{\vec{e}}$ для всех $n$ ребер $\Gamma(X)$.

Простейшей иллюстрацией к теореме 1.2 могут служить пунктированные кривые, двойственный граф которых является деревом, а все неприводимые компоненты - рациональными кривьми. Стабильность такой кривой $X$ по Делиню-Мамфорду равносильна тому, что валентность каждой вершины двойственного графа $\Gamma(X)$ не менее трех, что, в свою очередь, эквивалентно тому, что th $\Gamma(X) \geqslant 3$. Следовательно, согласно теореме 1.2 каноническое отображение в этом случае является вложением в проективное пространство размерности $g_{\gamma}-1=n-2$, где

\footnotetext{
${ }^{2}$ После сдачи этой работы в печать автор узнал, что аналогичные результаты для компактных (т.е. не пунктированных) кривых получены в работе F. Catanese, M. Franciosi, K. Hulek, M. Ried "Embeddings of curves and surfaces" (Nagoya Math. J. 1999. V. 154. P. 185-220).
} 

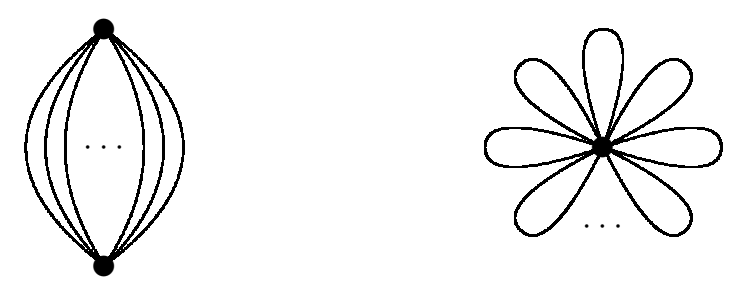

Рис. 4. Двойственные графы обобшенных гиперэллиптических кривых

через $n$ обозначено число параболических ребер графа $\Gamma(X)$. Образ канонического вложения - кривая степени $n-2$ - является, следовательно, кривой Веронезе или ее вырождением. При этом образы отмеченных точек представляют собой $n$ точек в проективном пространстве $\mathbb{P}^{n-2}$ в общем положении (последнее следует, например, из предложения 4.1) и, следовательно, многообразие модулей $\overline{\mathscr{M}}_{0, n}$ таких кривых представляет собой замыкание многообразия модулей кривых Веронезе в $\mathbb{P}^{n-2}$, проходящих через $n$ точек в обшем положении. Это гладкие рациональные многообразия размерности $n-3$, представляющие собой результат последовательного раздутия проективного пространства $\mathbb{P}^{n-3}$ в $n-1$ точке обшего положения, затем во всех соединяющих эти точки прямых, затем в плоскостях и т.д. до линейных подмногообразий коразмерности 2 (см., например, [4] или [1]). В частности, $\overline{\mathscr{M}}_{0,4}$ представляет собой проективную прямую, параметризуюшую пучок коник на плоскости, проходящих через четыре точки в общем положении, а $\overline{\mathscr{M}}_{0,5}$, параметризующая пространственные кубики, проходящие через пять точек в пространстве в общем положении, - поверхность дель Пещц степени пять. При этом исключительные прямые на этой поверхности соответствуют кубикам, распавшимся на плоскую конику и прямую, а точки их пересечения - кубикам, распавшимся на три прямые.

Удобно ввести на множестве модулярных графов отношение частичного порядка: $\Gamma^{\prime}>\Gamma$, если граф $\Gamma^{\prime}$ получен из графа $Г$ стягиванием некоторых его ребер. С каждым деревом $\Delta \mathrm{c} n$ параболическими ребрами связано открыто-замкнутое подмногообразие $\mathscr{M}_{\Delta} \subset \overline{\mathscr{M}}_{0, n}$, состоящее из пунктированных кривых, двойственный модулярный граф которых совпадает с $\Delta$. При этом отношение примыкания совпадает с введенньм отношением порядка на графах: $\mathscr{M}_{\Delta} \subset \overline{\mathscr{M}}_{\Delta^{\prime}}$, если $\Delta^{\prime}>\Delta$. Максимальному графу, имеющему одну вершину и $n$ параболических ребер, соответствует открытое множество неприводимых кривых Веронезе $\mathscr{M}_{0, n} \subset \overline{\mathscr{M}}_{0, n}$.

В заключение мы воспроизведем описание модулярной конфигурации из книги А.Н. Тюрина [5], опирающееся на развитую здесь технику. В [5] рассматриваются м-кривые, т.е. компактные кривые, имеющие трехвалентный двойственный граф̆ и рациональные неприводимые компоненты. Такие кривые, очевидно, не имеют модулей, поэтому однозначно определяются заданием своего двойственного графа Г. Все м-кривые стабильны по Делиню-Мамфорду и потому представляют собой конечную конфигурацию точек в компактификации многообразия модулей $\overline{\mathscr{M}}_{g}$ кривых рода $g$, параметризованную всевозможными трехвалентными графами рода $g$. 
В случае, когда двойственный граф м-кривой имеет толщину не менее трех, канонический образ такой кривой представляет собой конфигурацию $2 g-2$ прямых в проективном пространстве $\mathbb{P}^{g-1}$.

Рассмотрим теперь более общую кривую $X$, имеющую по-прежнему только рациональные неприводимые компоненты и двойственный граф $\Gamma=\Gamma(X)$, имеющий th $\Gamma \geqslant 3$, и предположим, что все его вершины, кроме одной, трехвалентны, а одна вершина $v_{0}$ имеет валентность $n \geqslant 3$, не имеет петель и при удалении ее граф сохраняет связность. Рассмотрим подграф $\Delta \subset \Gamma$, состоящий из единственной вершины $v_{0}$ и $n$ параболических ребер, соединявших $v_{0}$ с остальной частью графа $Г$. Модулярному графу $\Delta$ соответствует пунктированная кривая $Y$, представляющая собой просто компоненту $X_{v_{0}}$, на которой отмеченными точками объявлены все точки ее пересечения с остальными компонентами кривой $X$. Тогда из леммы 4.1 следует, что ограничение канонического отображения $\varphi_{X}$ кривой $X$ на кривую $Y$ совпадает с каноническим отображением кривой $Y$, которое, как мы видели, является вложением в $\mathbb{P}^{n-2}$. Аналогично, то же самое будет верно и для любой кривой $X^{\prime}$, двойственный граф которой $\Gamma\left(X^{\prime}\right)$ получается из графа $Г$ вклейкой вместо $v_{0}$ любого модулярного графа $\Delta^{\prime}<\Delta$ с $n$ параболическими ребрами, что дает нам вложение семейства $\overline{\mathscr{M}}_{0, n}$ в $\overline{\mathscr{M}}_{g}$. Очевидно, описанньм способом получаются все стабильные по Делиню-Мамфорду графы $\Gamma^{\prime}<\Gamma$, поэтому это семейство естественно обозначать $\overline{\mathscr{M}}_{\Gamma}$. При этом отношение примыкания таких многообразий $\overline{\mathscr{M}}_{\Gamma}$ индуцировано отношением порядка на графах Г. Это и есть модулярная конфигурация, описанная в девятой главе книги [5].

Теорема 1.3 (А.Н. Тюрин [5]). Компактификация Делиня-Мамфорда многообразия модулей $\overline{\mathscr{M}}_{g}$ кривых рода g содерәст конфигурачию рациональных подмногообразий $\mathscr{M}_{\Gamma}$, изоморфных $\overline{\mathscr{M}}_{0, n}$, параметризованных такими графами Г рода g толщины не менее трех, имеющими одну п-валентную и остальные трехвалентные вериины, что Г не теряет связности при выбрасывании n-валентной вершины. При этом отношение примыкания многообразий $\mathscr{M}_{\Gamma}$ индуцировано отношением порядка, так что $\mathscr{M}_{\Gamma} \cap \mathscr{M}_{\Gamma^{\prime}}=\mathscr{M}_{\min \left(\Gamma, \Gamma^{\prime}\right)}$.

Нетрудно заметить, что многообразия $\mathscr{M}_{\Gamma}$, проходящие через данную м-кривую $X$, соответствуют таким индуцированным ${ }^{3}$ подграфам $\Delta$ графа $\Gamma(X)$, которые являются деревьями, причем граф $\Gamma(X) \backslash \Delta$ связен.

При $n=3$ теорема дает просто конфигурацию конечного числа точек в $\overline{\mathscr{M}}_{g}$, соответствующих м-кривьм. При $n=4$ и $n=5$ условие связности автоматически следует из ограничения на толщину, что дает, соответственно, конфигурацию рациональных кривых и поверхностей дель Пецц в $\overline{\mathscr{M}}_{g}$, называемых в [5] специальньми однопараметрическими и специальными двупараметрическими деформациями м-кривых.

СлЕДСТВИЕ 1.1. Компактификачия Делиня-Мамфорда многообразия модулей $\overline{\mathscr{M}}_{g}$ кривых рода g содерэит конфигурацию рациональных кривых $\mathscr{M}_{\Gamma}$, параметризованных графами Г рода g толщины не менее трех, имеющими одну четырехвалентную и остальные трехвалентные вериины. Каждая такая кривая проходит ровно через три точки, соответствующие м-кривым,

\footnotetext{
${ }^{3}$ Подграф $\Delta \subset \Gamma$ назьвается индуцированньм, если он содержит все ребра графа $\Gamma$, соединяющие вершины подграфиа $\Delta$.
} 
и через каждуую точку, соответствующую м-кривой, проходит ровно $3 g-3$ таких кривых, находящихся во взаимно однозначном соответствии с множеством ребер двойственного графа м-кривой.

СлеДСТВИЕ 1.2. Компактификация Делиня-Мамфорда многообразия модулей $\overline{\mathscr{M}}_{g}$ кривых рода g содержит конфигурацию поверхностей дель Пецио степени пять $\mathscr{M}_{\Gamma}$, параметризованных графами Г рода g толщины не менее трех, имеющими одну пятивалентную вершину без петли и остальные трехвалентные вершины. Любъе две такие поверхности пересекаются только по исключительным прямым, представляющим собой многообразия $\mathscr{M}_{\Gamma^{\prime}}$, соответствующие графам $\Gamma^{\prime}<\Gamma$, имеющим одну четырехвалентную вериину (см. предыдущее следствие). Такие поверхности дель Пеццо, проходящие через точку, соответствующую данной м-кривой, находятся во взаимно однозначном соответствии с парами смежсных ребер двойственного графа, не включающихся в треугольник.

Эта работа, посвященная памяти моего учителя, Андрея Николаевича Тюрина, была начата осенью 2002 года под его руководством. Предварительный вариант был написан во время моего пребывания в Институте Макса Планка в Бонне. Пользуюсь случаем выразить Институту мою признательность за гостеприимство и стимулирующую рабочую обстановку. Я благодарю Ю.И. Манина за полезные обсуждения и С. К. Ландо за помощь в подготовке предварительного варианта этой работы.

\section{§ 2. Модулярные графы}

Для наших целей удобнее всего определять модулярный граф̆ следуюшим набором данных: $\left(V, \vec{E}, i, \vec{E}_{-}, s\right)$, где:

(1) $V$-множество вершин графа $\Gamma$;

(2) $\vec{E}$-множество ориентированных ребер графа $Г$;

(3) $i: \vec{E} \rightarrow \vec{E}-$ инволюиия смены ориентации (не имеющая неподвижных точек);

(4) $\vec{E}_{-}-$множество исходящих ориентированных ребер графа Г; требуется, чтобы $\vec{E}=\overrightarrow{E_{-}} \cup i\left(\overrightarrow{E_{-}}\right)$(т.е. каждое ориентированное ребро является входящим или исходящим);

(5) $s: \vec{E}_{-} \rightarrow V$ - сюръективное отображение, сопоставляющее каждому исходящему ребру его начало.

Отметим, что если $\vec{E}=\vec{E}$, то $\Gamma$ является обычным графом, каждое ребро которого имеет два конца. Такие графы мы будем называть компактными графами. В обшем же случае $\overrightarrow{E_{-}}$можно интерпретировать как множество ростков исходящих ребер, $\vec{E}_{+}=i\left(\vec{E}_{-}\right)-$как множество ростков входящих ребер, так что отображение $t=s \circ i: \overrightarrow{E_{+}} \rightarrow V$ сопоставляет каждому входящему ребру его конец. Звездой ростков исходящих из вершины $v \in V$ ребер называется множество

$$
S_{-}(v)=s^{-1}(v)
$$


Коэффициент инцидентности вершины $v$ и ориентированного ребра $\vec{e}$ определяется как

$$
\operatorname{inc}(\vec{e}, v)= \begin{cases}1, & \text { если } v=t(\vec{e}), \\ -1, & \text { если } v=s(\vec{e}), \\ 0 & \text { в остальных случаях. }\end{cases}
$$

Множество неориентированных ребер модулярного графа Г определяется как фактормножество $E=\vec{E} / i$; имеется естественная проекция “забывания ориентации" $\pi: \vec{E} \rightarrow E$. Количество элементов в множестве $S_{-}(v)$ называется валентностью вершины $v$.

Морфизмом $f$ из модулярного графа $\Gamma^{\prime}=\left(V^{\prime}, \overrightarrow{E^{\prime}}, i^{\prime}, \vec{E}_{-}^{\prime}, s^{\prime}\right)$ в модулярный граф $\Gamma=\left(V, \vec{E}, i, \overrightarrow{E_{-}}, s\right)$ называется пара отображений $f_{V}: V^{\prime} \rightarrow V$ и $f_{E}: \overrightarrow{E^{\prime}} \rightarrow \vec{E}$ таких, что $f_{E}\left(\overrightarrow{E_{-}^{\prime}}\right) \subseteq \vec{E}_{-}, f_{V} s^{\prime}=s f_{E}$ и $f_{E} i=i^{\prime} f_{E}$. Модулярный граф $\Gamma^{\prime}=$ $\left(V^{\prime}, \vec{E}^{\prime}, i^{\prime}, \overrightarrow{E_{-}^{\prime}}, s^{\prime}\right)$ называется подграфом графа $\Gamma=\left(V, \vec{E}, i, \overrightarrow{E_{-}}, s\right)$, если $V^{\prime} \subseteq V$, $\overrightarrow{E^{\prime}} \subseteq \vec{E}, \overrightarrow{E_{-}^{\prime}} \subseteq \vec{E}_{-}$и отображения $s^{\prime}$ и $i^{\prime}$ являются ограничениями отображений $s$ и $i$ на $\overrightarrow{E_{-}}$и $\vec{E}$. Таким образом, подграф $\Gamma^{\prime}$ модулярного графа $\Gamma$ определяется двумя подмножествами $V^{\prime} \subseteq V$ и $\overrightarrow{E^{\prime}} \subseteq \vec{E}$. Подграф $\Gamma^{\prime}=\left(V^{\prime}, \overrightarrow{E^{\prime}}\right)$ модулярного графа Г называется индуцированным, если дополнения $V^{\prime \prime}=V \backslash V^{\prime}$ и $\vec{E}^{\prime \prime}=$ $\vec{E} \backslash \vec{E}$ также определяют некоторый подграф $\Gamma^{\prime \prime}=\left(V^{\prime \prime}, \vec{E}^{\prime \prime}\right)$ модулярного графа $\Gamma$. В этом случае мы будем говорить, что $Г$ разбит на два индуцированных подграфа $\Gamma^{\prime}$ и $\Gamma^{\prime \prime}$, что эквивалентно тому, что

$$
\overrightarrow{E^{\prime}} \supset \vec{E} \backslash\left(s^{-1}\left(V^{\prime \prime}\right) \cup i\left(s^{-1}\left(V^{\prime \prime}\right)\right)\right)
$$

Если последнее включение является равенством, мы будем говорить, что индуцированный подграф $\Gamma^{\prime}$ является замкнутым подграфом графа $Г$; дополнительньй подграф̆ $\Gamma^{\prime \prime}$ мы будем тогда называть открытым подграфом графа $Г$.

Таким образом, разбиение графа Г на открытый и замкнутый подграфы задается разбиением множества вершин на два подмножества $V=V^{\prime} \cup V^{\prime \prime}, V^{\prime} \cap V^{\prime \prime}=\varnothing$; соответствующее разбиение множества ориентированных ребер задается тогда следующим образом:

$$
\vec{E}^{\prime \prime}=\left(s^{-1}\left(V^{\prime \prime}\right) \cup i\left(s^{-1}\left(V^{\prime \prime}\right)\right)\right), \quad \overrightarrow{E^{\prime}}=\vec{E} \backslash \overrightarrow{E^{\prime \prime}} .
$$

Морфизм $f$ модулярного графа $\Gamma^{\prime}$ в модулярный граф $Г$ будем называть звезднылм морфизмом, если ограничение отображения $f_{E}$ на звезду ростков ребер, исходящих из любой вершины $v^{\prime} \in V^{\prime}$, является взаимно однозначным соответствием

$$
f_{E}: S_{-}\left(v^{\prime}\right) \stackrel{\sim}{\rightarrow} S_{-}\left(f_{V}(v)\right)
$$

Отметим, что морфизм включения подграффа $\Gamma^{\prime}$ в графф $Г$ является звездным морфизмом тогда и только тогда, когда $\Gamma^{\prime}$ является открытым подграфом $\Gamma$.

Перейдем теперь к определению “звездны $x$ ” гомологий и когомологий модулярных графов, которые мы будем обозначать ${ }_{s} H$, чтобы отличать их от обычных 
топологических. Зафиксируем абелеву группу $A$ и произвольную ориентацию на множестве ребер графа Г, т.е. отображение

$$
\sigma: E \rightarrow \vec{E}
$$

сопоставляющее каждому ребру одну из двух его возможных ориентаций, так что $\pi \circ \sigma=\mathrm{Id}_{E}$. Мы опустим стандартную проверку того, что все последующие определения на самом деле не зависят от выбора ориентации $\sigma$.

Кограничный оператор $\delta$ из группы 1-коцепей $A^{E}$ в группу 2-коцепей $A^{V}$ :

$$
\delta: A^{E} \rightarrow A^{V}
$$

определяется формулой

$$
\delta\left(\left\{a_{e}\right\}\right)_{v}=\sum_{\vec{e} \in S_{-}(v)} \operatorname{inc}(\sigma(\pi(\vec{e})), v) \cdot a_{\pi(\vec{e})}
$$

так что

$$
{ }_{s} H^{1}(\Gamma, A)=\operatorname{Ker} \delta, \quad{ }_{s} H^{2}(\Gamma, A)=\operatorname{Coker} \delta .
$$

Аналогично, граничный оператор

$$
d: A^{V} \rightarrow A^{E}
$$

определяется формулой

$$
d(v)=\sum_{\vec{e} \in S_{-}(v)} \operatorname{inc}(\sigma(\pi(\vec{e})), v) \cdot \pi(\vec{e})
$$

и тогда

$$
{ }_{s} H_{1}(\Gamma, A)=\text { Coker } d, \quad{ }_{s} H_{2}(\Gamma, A)=\operatorname{Ker} d .
$$

Нетрудно проверить, что звездные гомологии и когомологии функториальны относительно звездных морфизмов модулярных графов:

ПРЕДЛОЖЕНИЕ 2.1. Пусть

$$
f: \Gamma^{\prime} \rightarrow \Gamma
$$

- звездный морфизм модулярных графов. Тогда имеются естественные гомоморфизмиц

$$
f^{*}:{ }_{s} H^{i}(\Gamma, A) \rightarrow{ }_{s} H^{i}\left(\Gamma^{\prime}, A\right)
$$

$u$

$$
f_{*}:{ }_{s} H_{i}\left(\Gamma^{\prime}, A\right) \rightarrow{ }_{s} H_{i}(\Gamma, A) .
$$

Нам также потребуется следуюшее несложное утверждение: 
ПРЕДЛОЖЕНИЕ 2.2. Пусть $f: \Gamma^{\prime} \rightarrow \Gamma-$ звездный морфизм и отображсение $f_{V}: V^{\prime} \rightarrow V$ сюрвективно. Тогда гомоморфизм

$$
f^{*}:{ }_{s} H^{1}(\Gamma, A) \rightarrow{ }_{s} H^{1}\left(\Gamma^{\prime}, A\right)
$$

ингективен.

Отметим, что для компактных модулярных графоо звездные гомологии и когомологии совпадают с обычньми симплициальными гомологиями (с заменой размерностей 2 на 0 и 0 на 2). Однако для модулярных графов с параболическими ребрами наши конструкции не сводятся к чисто топологическим. Для интуитивной мотивировки наших определений заметим, что над полем комплексных чисел кривые с простейшими двойными особенностями можно рассматривать как вырождения римановых поверхностей, полученные следующим образом. Зафиксируем на римановой поверхности $\Sigma$ несколько непересекающихся нестягиваемых окружностей $C_{e}, e \in E$, разбивающих поверхность на части $T_{v}, v \in V$, каждая из которых гомеоморфна римановой сфере с $k_{v}>1$ дырками. Стягивая каждую окружность в точку, мы получим (по крайней мере, топологически) нашу кривую $X_{\Sigma}$, двойственньй модулярный графф которой $\Gamma=\Gamma\left(X_{\Sigma}\right)$ имеет $V$ в качестве множества вершин и $E$ в качестве множества ребер. Зафиксируем ориентацию на поверхности $\Sigma$. Тогда выбор ориентации на каждой окружности $C_{e}$ эквивалентен выбору ориентации на соответствуюшем ребре $е$ графа Г. Линейная комбинация вершин $\sum a_{v} \cdot v$ графа $\Gamma$, которую мы назвали звездной 2-цепю, соответствует обычной 2 -цепи $\sum a_{v} \cdot T_{v}$ на поверхности $\Sigma$, а ее граница в гомологиях поверхности $\Sigma$ вычисляется в точности по формуле для граничного оператора в звездных гомологиях (2.11) модулярного граф̆а Г. Таким образом, мы получаем вложение

$$
{ }_{s} H_{1}(\Gamma, A) \hookrightarrow H_{1}(\Sigma, A)
$$

и изоморфиизм

$$
{ }_{s} H_{2}(\Gamma, A) \cong H_{2}(\Sigma, A) .
$$

Набор индексов пересечения произвольного цикла из $H_{1}(\Sigma, A)$ с окружностями $C_{e}$ является 1-коцилом из ${ }_{s} H^{1}(\Gamma, A)$, что задает гомоморфизм

$$
H_{1}(\Sigma, A) \rightarrow{ }_{s} H^{1}(\Gamma, A) .
$$

Если каждая из частей $T_{v}$ является сферой (с дырками), последовательность

$$
0 \rightarrow{ }_{s} H_{1}(\Gamma, A) \rightarrow H_{1}(\Sigma, A) \rightarrow{ }_{s} H^{1}(\Gamma, A) \rightarrow 0
$$

точна. Таким образом, звездные гомологии и когомологии Г получаются соответственно из А- и В-циклов на римановой поверхности $\Sigma$.

Обозначим через $R(\Gamma)$ множество компактных компонент модулярного графа $\Gamma$. Нетрудно проверить следующее утверждение.

ПРЕДЛОЖЕНИЕ 2.3.

$$
{ }_{s} H^{2}(\Gamma, A) \cong A^{R(\Gamma)}
$$




\section{СлЕДСТВИЕ 2.1.}

$$
{ }_{s} h^{1}(\Gamma)=|E|-|V|+|R| .
$$

Рассмотрим какой-нибудь компактный (не обязательно индуцированный) подграф̆ $\Gamma^{\prime}=\left(V^{\prime}, E^{\prime}\right)$ графа $\Gamma$; положим $V^{\prime \prime}=V \backslash V^{\prime}, E^{\prime \prime}=E \backslash E^{\prime}$. Рассмотрим коммутативную диаграмму

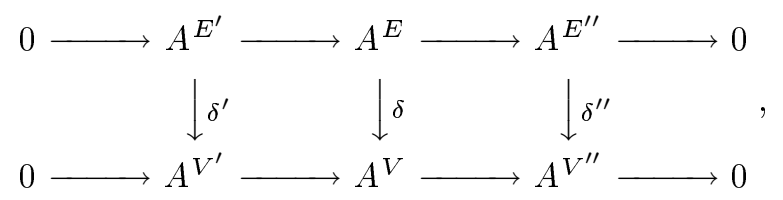

вертикальные стрелки в которой задаются формулой (2.8) (с заменой соответственно $E$ и $V$ на $E^{\prime}$ и $V^{\prime}$ или $E^{\prime \prime}$ и $\left.V^{\prime \prime}\right)$. Отсюда стандартным образом получается точная последовательность пары:

$$
\begin{aligned}
0 & \rightarrow{ }_{s} H^{1}\left(\Gamma^{\prime}, A\right) \rightarrow{ }_{s} H^{1}(\Gamma, A) \rightarrow{ }_{s} H^{1}\left(\Gamma, \Gamma^{\prime}, A\right) \\
& \rightarrow{ }_{s} H^{2}\left(\Gamma^{\prime}, A\right) \rightarrow{ }_{s} H^{2}(\Gamma, A) \rightarrow{ }_{s} H^{2}\left(\Gamma, \Gamma^{\prime}, A\right) \rightarrow 0 .
\end{aligned}
$$

Если компактньй подграф $\Gamma^{\prime}$ является индуцированным (и тем самњм замкнутьм), правый столбец (2.22) дает звездные когомологии графа $\Gamma^{\prime \prime}=\left(V^{\prime \prime}, E^{\prime \prime}\right)$. Используя предложение 2.3 , получаем следуюшее утверждение:

ПРЕДЛОЖЕНИЕ 2.4. Пусть Г" - открытый подграф модулярного графа Г, $\Gamma^{\prime}-$ дополнительный замкнутый подграф,

$$
i: \Gamma^{\prime \prime} \rightarrow \Gamma
$$

- отображение вложения. Тогда естественное отображение

$$
i^{*}:{ }_{s} H^{1}(\Gamma, A) \rightarrow{ }_{s} H^{1}\left(\Gamma^{\prime \prime}, A\right)
$$

включается в точную последовательность

$$
\begin{aligned}
0 & \rightarrow{ }_{s} H^{1}\left(\Gamma^{\prime}, A\right) \rightarrow{ }_{s} H^{1}(\Gamma, A) \stackrel{i^{*}}{\rightarrow} H_{s} H^{1}\left(\Gamma^{\prime \prime}, A\right) \\
& \rightarrow A^{R\left(\Gamma^{\prime}\right)} \rightarrow A^{R(\Gamma)} \rightarrow A^{R\left(\Gamma^{\prime \prime}\right)} \rightarrow 0
\end{aligned}
$$

СЛЕДСТВИЕ 2.2. Пусть в условиях предложения 2.4 каждая компактная компонента связности индуцированного замкнутого подграфа $\Gamma^{\prime}$ содержится в компактной компоненте связности графа Г, причем каждая компактная компонента графа Г содержит не более одной компактной связной компоненты подграфа $\Gamma^{\prime}$. Тогда имеется точная последовательность

$$
0 \rightarrow{ }_{s} H^{1}\left(\Gamma^{\prime}, A\right) \rightarrow{ }_{s} H^{1}(\Gamma, A) \stackrel{i^{*}}{\rightarrow}{ }_{s} H^{1}\left(\Gamma^{\prime \prime}, A\right) \rightarrow 0 .
$$


СлЕДСТВИЕ 2.3. Пусть в условиях предложения 2.4 графь $Г$ и $\Gamma^{\prime}$ связны. Если граф Г содержит параболические ребра, предположим дополнительно, что $\Gamma^{\prime}$ также содержит параболические ребра. Тогда имеется точная последовательность

$$
0 \rightarrow{ }_{s} H^{1}\left(\Gamma^{\prime}, A\right) \rightarrow{ }_{s} H^{1}(\Gamma, A) \stackrel{i^{*}}{\rightarrow} H^{1}\left(\Gamma^{\prime \prime}, A\right) \rightarrow 0
$$

Проследим, как меняются когомологии модулярного графа при выбрасывании из него некоторого подмножества $E^{\prime \prime} \subset E$ множества его ребер. Модулярньй граф̆ $\Gamma^{\prime}=\left(V, E \backslash E^{\prime \prime}\right)$ является компактньм (не обязательно индуцированным) подграфом графа $\Gamma$, и с помощью точной последовательности пары (2.23) нетрудно сравнить когомологии $Г$ и $\Gamma^{\prime}$. В этом случае ${ }_{s} H^{1}\left(\Gamma, \Gamma^{\prime}, A\right)=A^{E^{\prime \prime}}{ }_{\text {и }} H^{2}\left(\Gamma, \Gamma^{\prime}, A\right)=0$, так что мы получаем точную последовательность

$$
0 \rightarrow{ }_{s} H^{1}\left(\Gamma^{\prime}, A\right) \rightarrow{ }_{s} H^{1}(\Gamma, A) \rightarrow A^{E^{\prime \prime}} \rightarrow A^{R^{\prime}} \rightarrow A^{R} \rightarrow 0 .
$$

Тем самым доказано следующее утверждение:

ПРЕДЛОЖЕНИЕ 2.5. Пусть модулярный граф $\Gamma^{\prime}$ получается из модулярного графа $Г$ удалением некоторого множества ребер $E^{\prime \prime} \subset E$. Тогда имеется точная последовательность

$$
0 \rightarrow{ }_{s} H^{1}\left(\Gamma^{\prime}, A\right) \rightarrow{ }_{s} H^{1}(\Gamma, A) \rightarrow A^{E^{\prime \prime}} \rightarrow A^{\left|R^{\prime}\right|-|R|} \rightarrow 0
$$

Отметим, что число $\left|R^{\prime}\right|-|R|$ показывает, насколько увеличилось количество компактных связных компонент после удаления множества ребер $E^{\prime \prime}$. По определению толщины th(Г) модулярного граф̆а $\Gamma\left|R^{\prime}\right|-|R|=0$, если $\left|E^{\prime \prime}\right|<\operatorname{th}(\Gamma)$.

СлеДСТВИЕ 2.4. Если $\left|E^{\prime \prime}\right|<\operatorname{th}(\Gamma)$, то ребра из $E^{\prime \prime}$ налагают независимые условия на ${ }_{s} H^{1}(\Gamma, A)$.

Отметим важный частный случай, когда мы удаляем все параболические ребра граффа $\Gamma$, т.е. $E^{\prime \prime}=E_{\text {par }}$.

ПРЕДЛОЖЕНИЕ 2.6. Имеется точная последовательность

$$
0 \rightarrow{ }_{s} H^{1}\left(\Gamma_{\text {closed }}, A\right) \rightarrow{ }_{s} H^{1}(\Gamma, A) \rightarrow A^{E_{\mathrm{par}}} \rightarrow A^{R_{\mathrm{par}}} \rightarrow 0,
$$

правый гомоморфизм в которой задается формулой

$$
\sum_{e \in E_{\mathrm{par}}} a_{e} \cdot e \mapsto \sum_{r \in R_{\mathrm{par}}}\left(\sum_{e \in E_{\mathrm{par}}^{r}} a_{e}\right) \cdot r
$$

где через $R_{\mathrm{par}}$ обозначено множество связных компонент модулярного гра$\oint а \Gamma$, содержащих параболические ребра, а через $E_{\mathrm{par}}^{r}-$ множество параболических ребер компоненты $r \in R_{\mathrm{par}}$. 


\section{§ 3. Дифференциалы}

Рассмотрим пунктированную особую кривую с простейшими особенностями $X$, имеюшую двойственный модулярный граф̆ $\Gamma(X)$ с множеством вершин $V$, множеством ребер $E$ и множеством компактных компонент $R$. Сечение пучка регулярных дифференциальных форм $\Omega_{X}^{1}$ на $X$ представляет собой набор $\left\{\omega_{v}\right\}$ сечений ограничений подъемов $\nu^{*} \Omega_{X}^{1}$ на каждую компоненту $X_{v}^{\nu}$, которые, конечно, являются просто пучками дифференциалов третьего рода на $X_{v}^{\nu}$ с полюсами в точках $P_{\vec{e}}$, $\vec{e} \in S_{-}(v)$ :

$$
\left.\left(\nu^{*} \Omega_{X}^{1}\right)\right|_{X_{v}^{\nu}}=\Omega_{X_{v}^{\nu}}^{1}\left(\sum_{\vec{e} \in S_{-}(v)} P_{\vec{e}}\right) .
$$

Эти дифференциалы удовлетворяют условиям согласованности в каждой особой точке пересечения компонент $X_{v_{1}}$ и $X_{v_{2}}$ (возможно, совпадающих):

$$
\operatorname{res}_{P_{\vec{e}}} \omega_{v_{1}}+\operatorname{res}_{P_{\overleftarrow{e}}} \omega_{v_{2}}=0
$$

(Здесь и далее $\vec{e}$ и $\overleftarrow{e}$ обозначают две возможные ориентации ребра $e$, так что $s(\overleftarrow{e})=t(\vec{e})=v_{1}$ и $t(\overleftarrow{e})=s(\vec{e})=v_{2}$.) Заффиксируем некоторую ориентацию $\sigma$ (см. (2.6)) на ребрах графа $\Gamma(X)$. Тогда имеется отображение

$$
H^{0}\left(X, \Omega_{X}^{1}\right) \rightarrow k^{E}
$$

сопоставляющее каждому регулярному дифференциалу $\omega \in H^{0}\left(X, \Omega_{X}^{1}\right)$ набор его вычетов во всех его точках $p_{e}$ :

$$
\omega \mapsto\left\{\operatorname{res}_{P_{\sigma}(e)} \omega_{s(\sigma(e))}, e \in E\right\} .
$$

Уравнение (3.2) означает, что это отображение не зависит от выбора ориентации $\sigma$, а из основной теоремы о вычетах следует, что образ является коциклом. Тем самым, получается отображение

$$
H^{0}\left(X, \Omega_{X}^{1}\right) \rightarrow{ }_{s} H^{1}(\Gamma(X), k) .
$$

Это отображение сюръективно, поскольку на любой неособой кривой существует дифференциал третьего рода с любьм предписанньм набором значений (с нулевой суммой) в заданных точках (см. [6]). Ядро этого отображения состоит из дифференциалов, регулярных на каждом $X_{v}^{\nu}$.

ПРЕДЛОЖЕНИЕ 3.1. Имеется точная последовательность

$$
0 \rightarrow \bigoplus_{v \in V} H^{0}\left(X_{v}^{\nu}, \Omega_{X_{v}^{\nu}}^{1}\right) \rightarrow H^{0}\left(X, \Omega_{X}^{1}\right) \rightarrow{ }_{s} H^{1}(\Gamma(X), k) \rightarrow 0 .
$$

СлЕДСТвИЕ 3.1 .

$$
\operatorname{dim} H^{0}\left(X, \Omega_{X}^{1}\right)=\sum_{v \in V} g\left(X_{v}^{\nu}\right)+|E|-|V|+|R| .
$$


Мы будем обозначать эту размерность через $g_{\gamma}(X)$.

Теперь с помощью предложения 3.1 мы можем переписать точные последовательности из предыдушего параграффа, заменяя ${ }_{s} H^{1}(\Gamma(X), k)$ на $H^{0}\left(X, \Omega_{X}^{1}\right)$. Иногда удобно рассматривать пунктированную кривую $X$ как некомпактную кривую, полученную из компактной удалением всех отмеченных точек $p_{e}, e \in E_{\text {par. }}$. При этом кривая без отмеченных точек останется компактной; “забывание” всех отмеченных точек на пунктированной кривой $X$ соответствует переходу к компактификации кривой, которую мы будем обозначать через $X_{\text {closed }}$.

Используя (3.1) и (2.30), получаем точную последовательность:

$$
0 \rightarrow H^{0}\left(X_{\text {closed }}, \Omega_{X_{\text {closed }}}\right) \rightarrow H^{0}\left(X, \Omega_{X}^{1}\right) \rightarrow k^{E_{\text {par }}} \rightarrow k \rightarrow 0
$$

Средний гомоморфизм в этой последовательности сопоставляет каждому дифференциалу третьего рода набор его вычетов в отмеченных точках, а правый по-прежнему задается формулой (2.31). Конечно, полученная последовательность есть просто основная теорема о вычетах для пунктированных кривых.

ПРЕДЛОЖЕНИЕ 3.2. 1. Сумма вычетов регулярного дифференциала на пунктированной кривой во всех отмеченных точках равна нулю.

2. На пунктированной кривой существует регулярный дифференциал с любым предписанным набором вычетов в отмеченных точках (с нулевой суммой).

Рассмотрим индуцированный замкнутый подграф $\Gamma^{\prime}$ модулярного графа $\Gamma(X)$, обозначим через $\Gamma^{\prime \prime}$ дополнительный открытый подграф. Рассмотрим соответствующие им кривые:

$$
X^{\prime}=\bigcup_{v \in V^{\prime}} X_{v}, \quad X^{\prime \prime}=\bigcup_{v \in V^{\prime \prime}} X_{v} .
$$

Кривые $X^{\prime}$ и $X^{\prime \prime}$ наследуют все отмеченные точки кривой $X$, а $X^{\prime \prime}$ дополнительно приобретает новые отмеченные точки, а именно ветви особых точек пересечения с $X^{\prime}$ (см. (2.4)). Тогда $\Gamma^{\prime}=\Gamma\left(X^{\prime}\right)$ и $\Gamma^{\prime \prime}=\Gamma\left(X^{\prime \prime}\right)$. (Интерпретируя отмеченные точки как выколотые, можно считать, что мы просто отрываем компоненты $X^{\prime}$ от $X^{\prime \prime}$ таким образом, что точки пересечения $X^{\prime}$ с $X^{\prime \prime}$ отходят только к $X^{\prime}$, а на компонентах $X^{\prime \prime}$ на их месте остаются проколы.)

Комбинируя (3.1) с (2.25), получаем следующую точную последовательность:

$$
\begin{aligned}
0 & \rightarrow H^{0}\left(X^{\prime}, \Omega_{X^{\prime}}^{1}\right) \stackrel{u}{\rightarrow} H^{0}\left(X, \Omega_{X}^{1}\right) \stackrel{q}{\rightarrow} H^{0}\left(X^{\prime \prime}, \Omega_{X^{\prime \prime}}^{1}\right) \\
& \rightarrow k^{R^{\prime}} \rightarrow k^{R} \rightarrow k^{R^{\prime \prime}} \rightarrow 0
\end{aligned}
$$

Здесь $R^{\prime}$ и $R^{\prime \prime}$ обозначают множества компактных компонент связности графов $\Gamma^{\prime}$ и $\Gamma^{\prime \prime}$, вложение $u$ продолжает регулярные дифференциальные формы из $H^{0}\left(X^{\prime}, \Omega_{X^{\prime}}^{1}\right)$ нулем на все $X$, а $q$ является отображением ограничения регулярных дифференциалов с $H^{0}\left(X, \Omega_{X}^{1}\right)$ на $X^{\prime \prime}$.

Используя следствие 2.2 , получаем следующее утверждение. 
ПРЕДЛОЖЕНИЕ 3.3. Пусть $X^{\prime}$ - замкнутое подмножество кривой $X$, состоящее из некоторых неприводимых компонент кривой $X$, положим $X^{\prime \prime}=$ $X \backslash X^{\prime}$. Предположим, что кажсдая компактная компонента связности кривой $X^{\prime}$ содержится в компактной компоненте связности кривой $X$, причем каждая компактная компонента связности кривой $X$ содержит не более одной компактной компоненты связности кривой $X^{\prime}$. Тогда любую регулярную дифференииальную форму на $X^{\prime \prime}$ можсно продолжсить до регулярной дифференииальной формы на $X$.

СлЕДСТВИЕ 3.2. Предположим, что $X^{\prime}$ является связным замкнутым подмножеством связной компактной кривой $X$, состоящим из некоторых неприводимых компонент кривой $X$, a $X^{\prime \prime}=X \backslash X^{\prime}$. Тогда любую регулярную дифференциальную форму на $X^{\prime \prime}$ можнно продоләсить до регулярной дифференииальной формын на $X$.

Рассмотрим теперь подграф $\Gamma^{\prime}$ графа $\Gamma(X)$, полученный выбрасыванием некоторого множества ребер $E^{\prime \prime} \subset E ; X^{\prime}$-пунктированная кривая, соответствующая $\Gamma^{\prime}$. Тогда имеется точная последовательность

$$
0 \rightarrow H^{0}\left(X^{\prime}, \Omega_{X^{\prime}}^{1}\right) \rightarrow H^{0}\left(X, \Omega_{X}^{1}\right) \rightarrow k^{E^{\prime \prime}} \rightarrow k^{\left|R^{\prime}\right|-|R|} \rightarrow 0 .
$$

СлеДСТВИЕ 3.3. Пусть $X-$ связная кривая, $E^{\prime \prime} \subset E$ - такое подмножество множества ребер графа $\Gamma(X)$, что $|R(\Gamma(X))|$ не увеличивается после удаления из графа $Г(X)$ всех ребер из $E^{\prime \prime}$. Тогда существует регулярный дифференциал на $X$ с любым предписанным набором значений вычетов в точкax $p_{e}, e \in E^{\prime \prime}$.

\section{§4. Каноническое отображение}

Рассмотрим каноническое отображение

$$
\varphi_{X}: X \rightarrow \mathbb{P}\left(H^{0}\left(X, \Omega_{X}^{1}\right)^{*}\right)=\mathbb{P}^{g_{\gamma}-1}
$$

где $g_{\gamma}=h^{0}\left(X, \Omega_{X}^{1}\right)$.

Выясним сначала роль отмеченных точек, сравнив канонические отображения

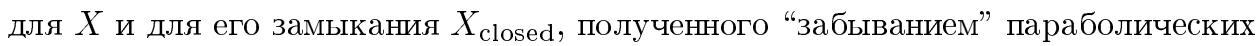
точек. Из точной последовательности (3.8) немедленно получается следующее утверждение.

ПРЕДЛОЖЕНИЕ 4.1. 1. Каноническое отображение замыкания $X_{\text {closed }}$ пунктированной кривой $X$ c $m>1$ отмеченной точкой является композичией канонического отображения кривой $X$ и проектирования из $(m-2)$-мерного проективного пространства, порохсенного точками $\varphi_{X}\left(p_{e}\right), e \in E_{\text {par. }}$.

2. Каноническое отобрахсение пунктированной кривой $X$ с одной отмеченной точкой совпадает с каноническим отображсением ее замыкания $X_{\text {closed. }}$

Пусть теперь на кривой $X$ имеется $2 m$ отмеченных точек, соответствующих $2 m$ параболическим ребрам

$$
e_{1}, \ldots, e_{m}, e_{1}^{\prime}, \ldots, e_{m}^{\prime}
$$


модулярного графа $\Gamma(X)$. Рассмотрим кривую $\widetilde{X}$, полученную из $X$ склеиванием каждой пары точек $p_{e_{l}}, p_{e_{l}^{\prime}}$ в двойную особую точку $p_{\tilde{e}_{l}}$ кривой $\widetilde{X}, l=1, \ldots, m$. Предположим для простоты, что кривая $X$ не имела компактных связных компонент (такие компоненты все равно никак не изменятся в результате нашей склейки) и что кривая $\widetilde{X}$ связна (в противном случае все рассуждения можно провести отдельно для каждой связной компоненты $\widetilde{X})$. Отображение склейки $\pi: X \rightarrow \widetilde{X}$ индуцирует звездный морфизм двойственных графов $\check{\pi}: \Gamma(X) \rightarrow \Gamma(\widetilde{X})$. С помощью предложений 3.1 и 2.2 получаем вложение $\check{\pi}^{*}:{ }_{s} H^{1}(\Gamma(\widetilde{X}), k) \hookrightarrow{ }_{s} H^{1}(\Gamma(X), k)$, являющееся просто подъемом дифференциальных форм с $\widetilde{X}$ на $X$. Сравнивая размерности в соответствии с (2.21), получаем точную последовательность

$$
0 \rightarrow H^{0}\left(\tilde{X}, \Omega_{\widetilde{X}}^{1}\right) \rightarrow H^{0}\left(X, \Omega_{X}^{1}\right) \rightarrow k^{d} \rightarrow 0
$$

где

$$
d= \begin{cases}m-1, & \text { если кривая } \widetilde{X} \text { компактна, } \\ m, & \text { если кривая } \widetilde{X} \text { не компактна. }\end{cases}
$$

ПРЕДЛОЖЕНИЕ 4.2. Каноническое отображение кривой $\widetilde{X}$, полученной из пунктированной кривой $X$ попарной склейкой $m$ пар отмеченных точек кривой $X$, является композичией канонического отображсения кривой $X$ u, при $d>0$, проектирования из $(d-1)$-мерного проективного пространства, где d задано формулой (4.4).

Очевидно, $d$ может оказаться нулем только если $m=1$ и кривая $\widetilde{X}$ компактна; в этом случае (4.3) является изоморфизмом.

СЛЕДСТВИЕ 4.1. Каноническое отображение пунктированной кривой $X$ с двумя отмеченными точками является композицией отображсения “склей$\kappa и " ~ \pi: X \rightarrow \widetilde{X}$ (которое является изоморфизмом вне отмеченных точек и отображает каждую из отмеченных точек в одну и ту же двойную особую точку) и канонического отображения кривой $\widetilde{X}$.

Предположим теперь, что в описываемой ситуации две отмеченные точки расположены на разных компонентах связности $X_{1}$ и $X_{2}$ кривой $X$. Тогда

$$
H^{0}\left(X, \Omega_{X}^{1}\right)=H^{0}\left(X_{1}, \Omega_{X_{1}}^{1}\right) \oplus H^{0}\left(X_{2}, \Omega_{X_{2}}^{1}\right)
$$

так что каноническое отображение кривой $X$ представляет собой пару канонических отображений кривых $X_{1}$ и $X_{2}$ в непересекающиеся проективные подпространства в $\mathbb{P}\left(H^{0}\left(X, \Omega_{X}^{1}\right)^{*}\right)$. Но как мы уже видели, это отображение является каноническим отображением связной кривой $\widetilde{X}$, которое, следовательно, не определено в точке $p_{\tilde{e}_{1}}$. Это, конечно, в точности случай толщины 1 , поскольку удаление из модулярного графа $\Gamma(\widetilde{X})$ ребра $\tilde{e}_{1}$ разбивает его на две компактные компоненты связности.

ПРЕДЛОЖЕНИЕ 4.3. 1. Пусть $X-$ связная компактная кривая $и$

$$
\operatorname{th}(\Gamma(X))=1
$$

Тогда канонический класс $\Omega_{X}^{1}$ не обилен. 
2. Пусть кривая $X$ представляет собой обвединение двух связных кривых $X_{1}$ и $X_{2}$, пересекающихся межсуу собой в одной двойной особой точке $p_{e}$. Тогда $p_{е}$ является базисной точкой канонического отображсения $\varphi_{X}$, а ограничение $\varphi_{X}$ на $X_{1}$ и $X_{2}$ представляет собой каноническое отображсение $X_{1}$ и $X_{2}$ в непересекающиеся проективнъе подпространства в $\mathbb{P}\left(H^{0}\left(X, \Omega_{X}^{1}\right)^{*}\right)$.

Тем самым, показана необходимость условия $\operatorname{th}(\Gamma(X)) \geqslant 2$ из первой части теоремы 1.2.

Рассмотрим теперь случай, когда двойственный модулярный граф компактной кривой $X$ имеет $\operatorname{th}(\Gamma(X))=2$. Предположим, что ребра $e_{1}$ и $e_{2}$ соединяют два связных подграфа $\Gamma\left(X_{1}\right)$ и $\Gamma\left(X_{2}\right)$, соответствующих двум кривым $X_{1}$ и $X_{2}$, пересекающимся в двух двойных особых точках $p_{e_{1}}$ и $p_{e_{2}}$ кривой $X=X_{1} \cup X_{2}$. Рассмотрим некомпактные кривые $X_{i}^{0}=X_{i} \backslash\left\{p_{e_{1}}, p_{e_{2}}\right\}, i=1,2$ (другими словами, мы отмечаем точки $p_{e_{1}}$ и $p_{e_{2}}$, неособые на кривых $X_{1}$ и $\left.X_{2}\right)$, так что графы $\Gamma\left(X_{1}^{0}\right)$ и $\Gamma\left(X_{2}^{0}\right)$ являются открытьми подграффами в $\Gamma(X)$, а $\Gamma\left(X_{2}\right)$ и $\Gamma\left(X_{1}\right)$ являются соответственно дополнительными замкнутыми подграфами. Несвязная кривая $X_{1}^{0} \sqcup X_{2}^{0}$ содержит две пары отмеченных точек, попарная склейка которых дает кривую $X$, поэтому согласно предложению 4.2 каноническое отображение $X$ является композицией канонического отображения $X_{1}^{0} \sqcup X_{2}^{0}$ и проектирования из точки. Но каноническое отображение кривой $X_{1}^{0} \sqcup X_{2}^{0}$ представляет собой просто пару канонических отображений кривых $X_{1}^{0}$ и $X_{2}^{0}$. Согласно следствию 4.1 каноническое отображение каждой из кривых $X_{i}^{0}$ склеивает точки $p_{e_{1}}$ и $p_{e_{2}}$ на каждой $X_{i}^{0}$ и, следовательно, то же самое происходит при действии отображения $\varphi_{X}$. Тем самњм, доказана необходимость условия $\operatorname{th}(\Gamma(X)) \geqslant 3$ в теореме 1.2 .

ПРЕДЛОЖЕНИЕ 4.4. 1. Каноническое отображение связной компактной кривой $c \operatorname{th}(\Gamma(X))=2$ не является влохсением.

2. Пусть ребра е е и е еразбивают двойственный граф $Г(X)$ на два подгра$\oint a$, т.е. кривая $X$ является обғединением двух связных компактных кривых, пересекающихся в двух двойных особъх точках $p_{e_{1}}$ и $p_{e_{2}}$ (неособъх на кажсдой компоненте $X_{i}$ ). Тогда ограничение канонического отображсения $\varphi_{X} н а$ каждую $X_{i}, i=1,2$, является каноническим отображсением пунктированной кривой $X_{i}^{0}$, представляющей собой кривую $X_{i}^{0}$ с отмеченными точками $p_{e_{1}}$ и $p_{e_{2}}$. Отображсения $\varphi_{X_{1}^{0}}$ и $\varphi_{X_{2}^{0}}$ переводят кривые $X_{1}$ и $X_{2}$ в два проективных подпространства в $\mathbb{P}\left(H^{0}\left(X, \Omega_{X}^{1}\right)^{*}\right)$ с одной общей точкой $M$, и если $\varphi_{X_{1}^{0}}$ и $\varphi_{X_{2}^{0}}$ определенв в точках $p_{e_{1}}$ и $p_{e_{2}}, \operatorname{mo} \varphi_{X}\left(p_{e_{1}}\right)=\varphi_{X}\left(p_{e_{2}}\right)=M$.

Случай некомпактных кривых толщины 2 разбирается аналогично, но проще свести все к случаю компактной кривой (см. предложение 4.2).

Приведем теперь несложное индуктивное рассуждение, которое пригодится при доказательстве теоремы 1.2. Пусть $\Gamma^{\prime \prime}$ - некоторьй открытый подграф̆ двойственного графа $\Gamma(X)$ некоторой пунктированной кривой $X, X^{\prime \prime}=\bigcup_{v \in V^{\prime \prime}} X_{v}-$ соответствующая ему пунктированная кривая. Предположим, что дополнительный замкнутый подграф $\Gamma^{\prime}$ связен. Применяя следствие 3.2 , получаем следующее утверждение:

ЛЕмма 4.1. Пусть $\Gamma^{\prime \prime}$ - такой открытый подграф двойственного графа $\Gamma(X)$ некоторой пунктированной кривой $X$, что дополнительный замкну- 
тый подграф $\Gamma^{\prime}$ связен. Тогда ограничение канонического отображсеия $\varphi_{X}$ кривой $X$ на кривую $X^{\prime \prime}$ совпадает с каноническим отображением $\varphi_{X^{\prime \prime}} к р и$ вой $X^{\prime \prime}$ :

$$
\left.\varphi_{X}\right|_{X^{\prime \prime}}=\varphi_{X^{\prime \prime}}
$$

Рассмотрим теперь некомпактную пунктированную кривую $X$ с $m$ отмеченными точками. Случаи $m=1,2$ описаны в предложении 4.1 и следствии 4.1 , поэтому предположим, что $m \geqslant 3$. Рассмотрим одноточечную компактификацию $\widehat{\Gamma}$ модулярного графа $\Gamma(X)$, полученную добавлением к $V$ одной дополнительной вершины $v_{0}$, инцидентной всем параболическим ребрам графа $\Gamma(X)$. Очевидно,

$$
\operatorname{th}(\widehat{\Gamma})=\operatorname{th}(\Gamma(X))
$$

Соответствующая кривая $\widehat{X}$ получается добавлением к кривой рациональной компоненты $X_{v_{0}}$, пересекающей $X$ во всех отмеченных точках кривой $X$, так что $\widehat{X}=X \cup X_{v_{0}}$ и $\Gamma(\widehat{X})=\widehat{\Gamma} ;$ для наших рассуждений будет неважно, какую именно рациональную кривую из $\mathscr{M}_{0, m}$ мы при этом выбираем. Кривую $\widehat{X}$ мы будем называть однокомпонентной компактификацией кривой $X$. Модулярный граф $\Gamma(X)$ является открытым подграфом графа $\widehat{\Gamma}$, причем дополнительный замкнутый подграф̆ состоит из одной вершины $v_{0}$. Применяя точную последовательность (3.10), получаем следуюшее утверждение:

ЛЕмма 4.2. Пусть $X-$ пунктированная кривая, $\widehat{X}$-ее однокомпонентная компактификачия. Тогда

$$
H^{0}\left(X, \Omega_{X}^{1}\right)=H^{0}\left(\widehat{X}, \Omega_{\widehat{X}}^{1}\right)
$$

и ограничение канонического отображения $\varphi_{\widehat{X}}$ кривой $\widehat{X}$ на X совпадает с каноническим отображением $\varphi_{X}$ кривой $X$.

Теперь мы готовы доказать первую часть теоремы 1.2. Согласно предыдущей лемме достаточно доказать теорему только для компактной кривой $X$. Предположим, что $\operatorname{th}(\Gamma(X)) \geqslant 2, x$-произвольная точка $X$. Чтобы доказать, что точка $x$ не является базисной, достаточно указать регулярную дифференциальную форму на $X$, не обрашаюшуюся в нуль в точке $x$. Заметим сначала, что поскольку $\operatorname{th}(\Gamma(X)) \geqslant 2$, модулярный граф $\Gamma(X)$ остается связным после удаления любого ребра $e \in E$. Согласно следствию 3.3 это значит, что сушествует регулярный дифференциал с любым предписанным вычетом в точке $p_{e}$, так что особая точка не может оказаться базисной. Пусть теперь $x$ - неособая точка некоторой неприводимой компоненты $X_{v}$ кривой $X$. Если $X_{v}$ не рациональна, в качестве $\omega$ можно взять любую регулярную дифференциальную форму на $X_{v}^{\nu}$, не обращающуюся в нуль в точке $x$, и продолжить ее нулем на все остальные компоненты $X$. Предположим, что $X_{v}$ рациональна. Если в вершине $v$ имеется хотя бы одна петля $e$, можно выбрать координату $z$ на проективной прямой $X_{v}^{\nu}$ таким образом, чтобы ветви соответствуюшей $e$ особой точки самопересечения компоненты $X_{v}$ имели бы координаты 0 и $\infty$. Тогда в качестве $\omega$ можно взять дифференциал $d z / z$ на $X_{v}^{\nu}$, продолженный нулем на остальные компоненты $X$. Предположим теперь, что в вершине $v$ нет петель. 
Попробуем в этом случае применить индуктивное рассуждение леммы 4.1 для открытого подграфа $\Gamma^{\prime \prime}$, имеюшего одну вершину $v_{0}$ и ребра $S\left(v_{0}\right)$. Поскольку $S\left(v_{0}\right)$ содержит по крайней мере два ребра, можно опять выбрать координату $z$ на проективной прямой $X_{v}^{\nu}$ таким образом, чтобы соответствующие особые точки имели бы координаты 0 и $\infty$. Тогда в качестве $\omega$ можно опять взять дифференциал $d z / z$ на $X_{v}^{\nu}$. Согласно лемме 4.1 этот дифференциал можно продолжить на всю кривую $X$, если модулярный граф $\Gamma(X)$ не теряет связность при удалении $v_{0}\left(\right.$ и $\left.S\left(v_{0}\right)\right)$. Таким образом, нам осталось только разобрать случай, когда удаление $v_{0}$ разбивает $\Gamma(X)$ на несколько несвязных частей, одну из которых мы назовем $\Gamma_{1}$, а все остальные $-\Gamma_{2}$. Ребра, соединяющие $\Gamma_{1}$ с $v_{0}$, являются параболическими ребрами графа $\Gamma_{1}$, поэтому согласно предложению 3.2 на соответствующей кривой $X_{1}$ существует дифференциал с любым предписанным набором вычетов в этих точках. Условие $\operatorname{th}(\Gamma(X)) \geqslant 2$ означает, что число таких ребер не менее двух, поэтому мы снова можем при подходящем выборе координаты взять дифференциал $d z / z$ на $X_{v}^{\nu}$, продолжить его на $X_{1}$ аналогично предыдущему рассуждению, а затем продолжить его нулем на $X_{2}$.

Первое утверждение теоремы 1.2 доказано.

\section{§ 5. Неприводимые кривые}

В этом параграфе мы докажем основную часть (т.е. пп. II и III) теоремы 1.2 для неприводимых кривых. Начнем с компактных неприводимых кривых. Двойственньй модулярньй графф в этом случае состоит из одной вершины с несколькими петлями, соответствующими особым точкам кривой.

Сначала нам потребуется несколько общих соображений. Пусть имеется такая компактная кривая с простейшими двойными точками $X, \operatorname{th}(\Gamma(X)) \geqslant 3$, каноническое отображение $\varphi_{X}$ которой является вложением в $\mathbb{P}^{g_{\gamma}-1}$ степени

$$
d_{\gamma}=2\left(g_{\gamma}-1\right)
$$

где

$$
g_{\gamma}=\sum_{v \in V} g\left(X_{v}^{\nu}\right)+|E|-|V|+1
$$

(Для связной компактной кривой $|R|=1,\left|E_{\text {par }}\right|=0$.) Отметим на кривой $X$ две неособые точки $p_{1}$ и $p_{2}$, получим новую пунктированную кривую $X^{0}$ и новую компактную кривую $\widetilde{X}$, полученную из $X$ склейкой точек $p_{1}$ и $p_{2}$ в двойную особую точку с ветвями $p_{1}$ и $p_{2}$. Согласно предложению 4.1 и следствию 4.1 канонические отображения $\varphi^{0}$ для $X^{0}$ и $\widetilde{\varphi}$ для $\widetilde{X}$ совпадают, а каноническое отображение $\varphi_{X}$ является композицией $\varphi^{0}=\widetilde{\varphi}$ и проектирования из точки $\varphi^{0}\left(p_{1}\right)=\varphi^{0}\left(p_{2}\right)$. Следовательно, $\widetilde{\varphi}$ является изоморфизмом повсюду, кроме, возможно, точек $p_{1}$ и $p_{2}$. Поскольку

$$
H^{0}\left(X^{0}, \Omega_{X^{0}}^{1}\right)=H^{0}\left(X, \Omega_{X}^{1}\left(p_{1}+p_{2}\right)\right),
$$

образ $\varphi^{0}$ имеет степень $d_{\gamma}+2$, поэтому обе ветви кривой $\varphi^{0}(X)$ неособы и, следовательно, $\varphi^{0}=\widetilde{\varphi}$ является вложением. Мы доказали следующее полезное утверждение: 
ЛЕмма 5.1. Пусть связная компактная кривая $\widetilde{X}$ получена из связной компактной кривой $X$ склеиванием двух неособых точек в одну простейшую двойную особую точку (так что двойственный модулярный граф $\Gamma(X)$ получается из двойственного модулярного графа $Г(\widetilde{X})$ удалением одного ребра). Предположим, что $\operatorname{th}(\Gamma(X)) \geqslant 3$ и что каноническое отображение $\varphi_{X} к р и$ вой $X$ является вложением. Тогда каноническое отображение $\varphi_{\tilde{X}}$ кривой $\widetilde{X}$ также является вложением.

Поскольку, например, каноническое отображение неособой негиперэллиптической кривой рода $g>2$ является вложением, то же самое верно для соответствующих особых кривых.

СлЕДСТВИЕ 5.1. Каноническое отображение неприводимой компактной кривой $X$ рода $g>2$ с простейшими двойньцми особъми точками, нормализация которой негиперәллиптична, является вложением.

Рассмотрим теперь гиперэллиптическую кривую $X(g \geqslant 2)$ с одной простейшей двойной особой точкой, причем предположим, что ветви этой особой точки $p_{1}$ и $p_{2}$ не переводятся одна в другую гиперэллиптической инволюцией $i: X^{\nu} \rightarrow X^{\nu}$; это значит, что дивизор $p_{1}+p_{2}$ не является дивизором нулей никакой рациональной функции на $X^{\nu}$.

Каноническое отображение $\varphi_{X}$ задается полной линейной системой

$$
\left|\Omega_{X^{\nu}}^{1}\left(p_{1}+p_{2}\right)\right| .
$$

Для доказательства того, что оно является вложением, достаточно проверить, что для любых двух точек $a, b \in X^{\nu},\{a, b\} \neq\left\{p_{1}, p_{2}\right\}$,

$$
h^{0}\left(X^{\nu}, \Omega_{X^{\nu}}^{1}\left(p_{1}+p_{2}-a-b\right)\right)=h^{0}\left(X^{\nu}, \Omega_{X^{\nu}}^{1}\left(p_{1}+p_{2}\right)\right)-2 .
$$

Согласно двойственности Серра это равносильно тому, что $h^{0}\left(X^{\nu}, \mathcal{O}_{X^{\nu}}(a+b-\right.$ $\left.\left.p_{1}-p_{2}\right)\right)=0$, что, как мы видели, верно. Тогда, используя лемму 5.1, мы по индукции получаем утверждение II теоремы 1.2 для гиперэллиптических кривых (точнее, следовало бы сказать: “для негиперэллиптических особых кривых, нормализация которых является гиперэллиптической кривой” ).

ПРЕДЛОЖЕНИЕ 5.1. Каноническое отображсение неприводимой компактной особой кривой $X$, нормализация которой является гиперәллиптической кривой, является вложением, если какие-нибудь две ветви какой-нибудь двойной особой точки кривой $X$ не находятся в гиперәллиптической инволюuиu.

Аналогично доказываются соответствующие утверждения для случаев, когда нормализация кривой эллиптична или рациональна.

ПРЕДЛОЖЕНИЕ 5.2. Каноническое отображение неприводимой компактной особой кривой $X$, имеющей не менее двух двойных особъх точек, нормализация которой является әллиптической кривой, является вложением, если не существует әллиптической инволюиии, переставляющей ветви каждой двойной особой точки кривой $X$. 
ПРЕДЛОЖЕНИЕ 5.3. Каноническое отображение неприводимой компактной особой рациональной кривой $X$, имеющей не менее двух двойнъх особъх точек, является вложением, если не существует проективной инволючии, переставляющей ветви каждой двойной особой точки кривой $X$.

Оба эти утверждения доказываются совершенно аналогично предыдущему, но в качестве базы индукции нужно брать эллиптическую кривую с двумя двойными точками для предложения 5.2 и рациональную кривую с тремя двойными точками для предложения 5.3. Это будут плоские квартики с соответственно двумя или тремя двойными точками.

Если же ветви каждой особой точки кривой $X$ переставляются какой-нибудь инволюцией $i: X^{\nu} \rightarrow X^{\nu}$, то нетрудно проверить, что все регулярные дифференциалы на $X$ подняты с рациональной кривой $X^{\nu} / i$ с отмеченными точками, соответствующими двойным точкам $X$, так что каноническое отображение кривой $X$ является композицией двулистного накрытия факторизации по инволюции и канонического отображения пунктированной кривой $X^{\nu} / i$, которое является в точности отображением Веронезе (см., например, [4] или [6].) Таким образом, теорема 1.2 для компактных неприводимых кривых полностью доказана.

Для некомпактных кривых нам потребуется еще несколько общих лемм. Предположим, что, как и в лемме 5.1 , имеется компактная особая кривая $X, \operatorname{th}(\Gamma(X)) \geqslant 3$, каноническое отображение которой $\varphi_{X}$ является вложением в $\mathbb{P}^{g_{\gamma}-1}$ степени

$$
d_{\gamma}(X)=2\left(g_{\gamma}-1\right)
$$

где

$$
g_{\gamma}(X)=\sum_{v \in V} g\left(X_{v}^{\nu}\right)+|E|-|V|+1
$$

Отметив какие-нибудь три неособые точки $p_{1}, p_{2}, p_{3}$ кривой $X$, получим пунктированную кривую $X^{0}$. Тогда $g_{\gamma}\left(X^{0}\right)=g_{\gamma}(X)+2, d_{\gamma}\left(X^{0}\right)=d_{\gamma}(X)+2$ и согласно предложению 4.1 каноническое отображение кривой $X$ является композицией канонического отображения кривой $X^{0}$ и проектирования из прямой. Сравнивая степени, получаем, что эта прямая пересекает $\varphi_{X^{0}}\left(X^{0}\right)$ в точности в трех точках $\varphi_{X^{0}}\left(p_{i}\right), i=1,2,3$, причем эти точки неособы на $\varphi_{X^{0}}\left(X^{0}\right)$. Поскольку $\varphi_{X}$ является вложением, $\varphi_{X^{0}}$ также является вложением. Конечно, аналогично мы можем отмечать на $X^{0}$ и большее количество точек: каждая новая отмеченная точка увеличивает $d_{\gamma}$ и $g_{\gamma}$ на 1 и мы можем повторить те же рассуждения. Таким образом, доказана следующая лемма:

ЛЕмма 5.2. 1. Предположим, что пунктированная связная особая кривая $X^{0}$ получена из компактной связной кривой $X$ выделением трех неособъх точек $p_{i}, i=1,2,3$ (так что модулярный граф $\Gamma\left(X^{0}\right)$ получен из графа $\Gamma(X)$ добавлением трех параболических ребер). Предположсим, что $\operatorname{th}(\Gamma(X)) \geqslant 3$ u каноническое отображение $\varphi_{X}$ кривой $X$ является вложсением. Тогда каноническое отображсение $\varphi_{X^{0}}$ кривой $X^{0}$ также является вложсением. Образь точек $\varphi_{X^{0}}\left(p_{i}\right), i=1,2,3$, лежсат на проективной прямой и являются единственными точками пересечения этой прямой с образом $\varphi_{X^{0}}\left(X^{0}\right)$. 
2. Предположим, что пунктированная связная особая кривая $X^{0}$ получена из пунктированной связной особой кривой $X$, имеющей по крайней мере три отмеченные точки, выделением еще одной неособой точки $p$ (так что модулярный граф $\Gamma\left(X^{0}\right)$ получен из модулярного графа $\Gamma(X)$ добавлением параболического ребра). Предположим, что $\operatorname{th}(\Gamma(X)) \geqslant 3$ и каноническое отображсение $\varphi_{X}$ кривой $X$ является вложсением. Тогда каноническое отображение $\varphi_{X^{0}}$ кривой $X^{0}$ также является вложением.

СЛЕДСТВИЕ 5.2. Предположим, что пунктированная связная особая кривая $X^{0}$ получена из компактной связной кривой $X$ вылелением $m \geqslant 3$ неособых точек $p_{i}, i=1, \ldots, m$ (так что модулярный граф $\Gamma\left(X^{0}\right)$ получен из модулярного графа $Г(X)$ добавлением $m$ параболических ребер). Предположсим, что th $(\Gamma(X)) \geqslant 3$ и каноническое отображсение $\varphi_{X}$ кривой $X$ является вложением. Тогда каноническое отображсние $\varphi_{X^{0}}$ кривой $X^{0}$ также является влохсением. Образъ точек $\varphi_{X^{0}}\left(p_{i}\right), i=1, \ldots, m$, порохдают $(m-2)$-мерное проективное пространство $\mathbb{P}^{m-2}$ и являются единственными точками пересечения этого проективного пространства $\mathbb{P}^{m-2}$ с образом $\varphi_{X^{0}}\left(X^{0}\right)$.

Докажем теперь аналог леммы 5.1.

ЛЕмма 5.3. Предположим, что пунктированная связная особая кривая $\widetilde{X}$, имеющая по крайней мере три отмеченные точки, получена из пунктированной связной особой кривой $X$ склеиванием двух неотмеченных неособых точек в еще одну особую двойную точку (так что модулярныи граф $Г(X)$ получен из модулярного графа $Г(\widetilde{X})$ удалением ребра). Предположим, что $\operatorname{th}(\Gamma(X)) \geqslant 3$ и каноническое отображсение $\varphi_{X}$ кривой $X$ является вложением. Тогда каноническое отображение $\varphi_{\widetilde{X}}$ кривой $\widetilde{X}$ также является вложсением.

Для доказательства леммы сделаем две неособые точки $p_{1}$ и $p_{2}$ на $X$, которые склеиваются в особую точку на $\widetilde{X}$, отмеченными точками и обозначим полученную таким образом пунктированную кривую через $X^{0}$. Тогда

$$
g_{\gamma}(\widetilde{X})=g_{\gamma}\left(X^{0}\right)+1=g_{\gamma}(X)+2, \quad d_{\gamma}(\widetilde{X})=d_{\gamma}\left(X^{0}\right)=d_{\gamma}(X)+2
$$

и каноническое отображение $\varphi_{\widetilde{X}}$ является композицией канонического вложения (лемма 5.2) $\varphi_{X^{0}}$ и проектирования из точки. Следовательно, $\varphi_{\tilde{X}}$ является изоморфизмом по крайней мере вне точек $p_{1}$ и $p_{2}$. Каноническое вложение $\varphi_{X}$ является композицией $\varphi_{\tilde{X}}$ и проектирования из точки $\varphi_{\tilde{X}}\left(p_{1}\right)=\varphi_{\tilde{X}}\left(p_{2}\right)$, понижающего степень на 2. Поэтому эта точка является простой двойной особой точкой кривой $\varphi_{\widetilde{X}}(\widetilde{X})$, так что $\varphi_{\widetilde{X}}$ является вложением.

Пусть теперь имеется неособая неприводимая пунктированная кривая, имеющая по крайней мере три отмеченных точки. Тогда, очевидно, ее каноническое отображение является вложением. Используя лемму 5.3, мы теперь можем добавить любое число двойных особых точек.

СлЕДСТВИЕ 5.3. Каноническое отображение неприводимой пунктированной кривой, имеющей по крайней мере три отмеченные точки, является вложением. 


\section{§6. Доказательство основной теоремы}

Будем доказывать теорему 1.2 индукцией по числу неприводимых компонент компактной кривой $X$. Поскольку имеются специальные (а именно обобщенно гиперэллиптические) кривые с $|V|=1,2$, базой индукции может быть только случай $|V| \geqslant 3$, поэтому нам потребуется отдельное рассмотрение случая двух и трехкомпонентных кривых.

Для того чтобы доказать, что отображение приводимой кривой является вложением, достаточно проверить две вещи. Во-первых, нужно доказать, что ограничение нашего отображения на каждую неприводимую компоненту является вложением. Во-вторых, необходимо проверить, что отображение разделяет точки, расположенные на разных неприводимых компонентах кривой $X$. Заметим, что согласно следствию 3.3 каноническое отображение при $\operatorname{th}(\Gamma(X)) \geqslant 3$ разделяет особые точки, поэтому мы можем ограничиться рассмотрением неособых точек. Более того, нетрудно убедиться, что не возникает особенных проблем с нерациональньми компонентами и с рациональными компонентами, имеющими двойную точку самопересечения.

ЛЕмма 6.1. Каноническое отображение компактной кривой, модулярный граф которой имеет толщину по крайней мере 3 , различает точки, расположенные на двух разных неприводимых компонентах, если по крайней мере одна из них либо нерациональна, либо рациональна, но имеет двойную точку самопересечения.

Для доказательства леммы достаточно указать регулярный дифференциал на $X$, различающий данные две точки. В случае, когда одна из компонент нерациональна, в качестве такого дифференциала можно взять всюду регулярную дифференциальную форму на одной из этих компонент, продолженную нулем на все остальные компоненты. На рациональной компоненте с двойной особой точкой можно взять диффференциал $d z / z$ (координата $z$ снова выбрана так, чтобы ветвями особой точки были 0 и $\infty$ ) и продолжить его нулем на все остальные компоненты.

Теперь уже нетрудно свести проблему различения точек, расположенных на разных компонентах, к случаю, когда все компоненты рациональны. Пусть имеются две неособые точки, расположенные на компонентах $X_{i}, i=1,2$. Как мы только что видели, можно предположить, что обе эти компоненты рациональны и не имеют точек самопересечения. Пусть $X_{i}$ - какая-нибудь другая компонента, тогда согласно лемме 4.1 мы можем свести задачу к пунктированной кривой $X^{0}=X \backslash X_{i}$ (которая соответствует открытому подграфу $\left.\Gamma(X) \backslash v_{i}\right)$, поскольку ограничение канонического отображения кривой $X$ на $X^{0}$ совпадает с каноническим отображением кривой $X^{0}$. Далее, мы можем перейти к однокомпонентной компактификации (лемма 4.2) кривой $X^{0}$, имеющей уже по определению рациональную компоненту $X_{i}^{\prime}$.

СЛЕДСТВИЕ 6.1. Пусть Г - модулярный граф толщины 3 без параболических ребер. Предположим, что каноническое отображение любой компактной кривой $X^{\prime}$, имеющей только рациональные неприводимые компоненты, двойственный модулярный граф которой $\Gamma\left(X^{\prime}\right)$ совпадает $е \Gamma$, разделяет точки, располохсенные на разных неприводимых компонентах. Тогда каноническое отображсене любой кривой $X$, двойственный модулярный граф 
которой $\Gamma(X)$ совпадает $c \Gamma$, разделяет точки, располохсенные на разных неприводимых компонентах.

Рассмотрим теперь компактную кривую $X=X_{1} \cup X_{2}$ с двумя неприводимыми компонентами $X_{1}$ и $X_{2}$. Двойственньй модулярный граф имеет две вершины $v_{1}$ и $v_{2}$, соединенные по крайней мере тремя ребрами, поскольку $\operatorname{th}(\Gamma(X) \geqslant 3$. Каждая вершина вместе с исходящими из нее ребрами образует открытый подграф̆, причем дополнительный замкнутый подграф (т.е. другая вершина) связен. Тем самьм, выполнены условия леммы 4.1. Следовательно, ограничение канонического отображения $\varphi_{X}$ кривой $X$ на каждую неприводимую компоненту $X_{i}$ совпадает с каноническим отображением $\varphi_{X_{i}}$ компоненты $X_{i}$, рассматриваемой как пунктированная кривая, отмеченньми точками которой являются точки ее пересечения с другой компонентой. Но согласно следствию $5.3 \varphi_{X_{i}}$ являются вложениями.

ПРЕДЛОЖЕНИЕ 6.1. Ограничение канонического отображсения на каждую неприводимую компоненту компактной кривой, имеющей две неприводимые компоненты и двойственный модулярный граф толщины не менее трех, является вложсением.

Теперь нам осталось выяснить, ког да каноническое отображение различает точки, расположенные на разных неприводимых компонентах. Лемма 6.1 сводит эту задачу к случаю, когда обе компоненты рациональны и не имеют точек самопересечения. Следовательно, нам осталось разобрать только случай, когда кривая $X$ состоит из двух неособых рациональных компонент, пересекающихся в $m \geqslant 3$ точках. Ограничение канонического отображения на каждую компоненту является в этом случае отображением Веронезе в одно и то же проективное пространство $\mathbb{P}^{m-2}$. Известно, что две кривые Веронезе в $\mathbb{P}^{m-2}$, имеющие более $m$ общих точек, совпадают (см., например, [6]). Таким образом, каноническое отображение является либо вложением, либо имеет степень два. В последнем случае канонические образы $\varphi_{X_{1}}\left(X_{1}\right)$ и $\varphi_{X_{2}}\left(X_{2}\right)$ обеих компонент совпадают. Тогда $\varphi_{X_{2}}^{-1} \circ \varphi_{X_{1}}$ является изоморфизмом между $X_{1}$ и $X_{2}$, переводящим друг в друга ветви их точек пересечения. Очевидно, верно и обратное: из наличия такого изоморфизма следует совпадение канонических образов обеих компонент, поскольку на рациональной кривой дифференциал третьего рода однозначно определяется набором своих вычетов во всех особых точках.

Итак, мы миновали все исключительные случаи и можем начать индуктивное доказательство того, что каноническое отображение связной компактной кривой с не менее чем тремя неприводимьми компонентами и толщиной двойственного модулярного графа не менее трех является вложением. Сначала разберем случай кривой с ровно тремя неприводимыми компонентами, являющийся для нашего доказательства базой индукции. Пусть имеется такая кривая $X$. Используя лемму 5.1, мы можем, удаляя ребра из двойственного графа, сводить задачу к кривой с меньшим количеством двойных особых точек, по крайней мере до тех пор, пока толщина получаемого графа остается не менее трех. Нетрудно проверить, что действуя таким образом, мы в конце концов придем к одному из двух минимальных трехвершинных графов толшины три, изображенных на рис. 5 .

Первый из изображенных на рис. 5 граффов остается связным после удаления любой из трех его вершин. Согласно лемме 4.1 это означает, что ограничение канони- 

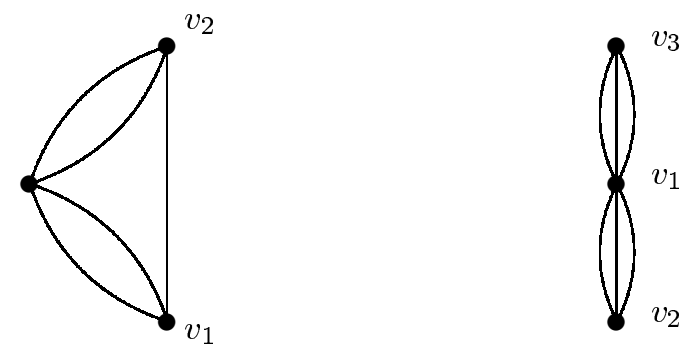

Р ис. 5. Два минимальных трехвершинных графа толщины 3

ческого отображения на каждую компоненту совпадает с каноническим отображением этой компоненты, рассматриваемой как пунктированная кривая, отмеченными точками которой являются точки ее пересечения с остальньми неприводимыми компонентами. Тогда согласно лемме 5.3 ограничение канонического отображения на каждую неприводимую компоненту является вложением и нам остается доказать, что каноническое отображение различает точки на разных неприводимых компонентах. Согласно следствию 6.1 мы при этом можем предположить, что все неприводимые компоненты этой кривой рациональны, но в этом случае канонический образ такой кривой представляет собой просто плоскую конику с двумя секушими в обшем положении.

Другой минимальный трехвершинный граф (“двойной тэта-граф”) на рис. 5 доставляет несколько большие трудности, поскольку он теряет связность при удалении средней вершины $v_{1}$. Аналогично предыдушему мы можем доказать, что ограничение канонического отображения на остальные две компоненты $X_{2}$ и $X_{3}$ является вложением, но для компоненты $X_{1}$, отвечаюшей средней вершине, требуется другое рассуждение.

Заметим, что если компонента $X_{1}$ нерациональна, каноническое отображение компактной двухкомпонентной кривой $X_{1} \cup X_{2}$ уже является вложением. Тогда согласно лемме 5.2 это же верно и для пунктированной кривой $X_{1} \cup X_{2}$, отмеченньми точками которой являются три точки пересечения с компонентой $X_{3}$. Но эта пунктированная кривая соответствует открытому подграфу двойственного граффа $\Gamma(X)$, причем дополнительный замкнутый подграф (т.е. вершина $v_{3}$ ) связен, поэтому согласно лемме 4.1 ограничение канонического отображения $\varphi_{X}$ на $X_{1} \cup X_{2}$ является вложением, что завершает доказательство для случая нерациональной компоненты $X_{2}$.

Пусть теперь компонента $X_{1}$ рациональна. Мы должны доказать, что ограничение канонического отображения $\varphi_{X}$ на $X_{1}$ является вложением и что каноническое отображение различает неособые точки, расположенные на компоненте $X_{1}$, от неособых точек, лежащих на компонентах $X_{i}, i=2,3$.

Первое доказательство мы опять можем свести к пунктированной двухкомпонентной кривой $X_{1} \cup X_{i}$ (лемма 4.1). Затем, переходя к однокомпонентной компактификации (лемма 4.2), свести задачу к случаю, когда третья компонента рациональна. Повторяя то же рассуждение для другой компоненты, мы сведем задачу к случаю, когда все неприводимые компоненты рациональны. Вторая из вышеупомянутых задач также сводится (см. лемму 6.1) к случаю, когда все компоненты рациональны. 
Канонический образ кривой с тремя рациональньми компонентами, двойственный граф̆ которой является “двойным тэта-граффом", описывается следующим образом. Выберем две тройки точек (все точки различны) на кривой Веронезе степени 4 в $\mathbb{P}^{4}$ и рассмотрим две плоскости, определенные каждой тройкой точек. Обе плоскости не имеют других общих точек с кривой Веронезе, поскольку в противном случае такая плоскость вместе с любой пятой точкой кривой порождала бы трехмерную гиперплоскость, пересекаюшую нашу кривую четвертой степени в пяти точках, что невозможно. Более того, если любая касательная или секущая кривой Веронезе пресечет одну из этих плоскостей, эта плоскость вместе с пересекающей ее прямой снова определяет трехмерную гиперплоскость, пересекаюшую нашу кривую четвертой степени в пяти точках, что невозможно. Эти две плоскости также не могут не находиться в общем положении, поскольку иначе они опять-таки определяют трехмерную гиперплоскость, пересекаюшую нашу кривую четвертой степени уже в шести точках, что также невозможно. Следовательно, эти две плоскости пересекаются ровно в одной точке. Проектирование из этой точки изоморфно отображает нашу кривую Веронезе на рациональную кривую степени 4 в $\mathbb{P}^{3}$, которая и является каноническим образом компоненты $X_{1}$. Две плоскости при этом переходят в две прямые, каждая из которых трансверсально пересекает $X_{1}$ ровно в трех точках. Эти прямые и являются каноническими образами компонент $X_{2}$ и $X_{3}$. Итак, случай трехкомпонентных кривых полностью разобран.

Отправляясь от “двойного тэта-графа”, мы можем доказать теорему 1.2 для любой компактной кривой $X$, двойственный модулярный граф̆ которой имеет $m+1$ вершину $v_{0}, v_{1}, \ldots, v_{m}$ и $3 m$ ребер, так что каждая вершина $v_{i}, i=1, \ldots, m$, соединена с $v_{0}$ тремя ребрами (“звездный тэта-граф.", рис. 6).

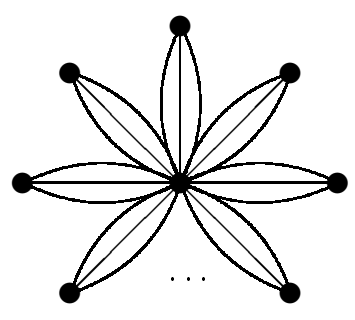

Рис. 6. “Звездный тэта-графф" - единственный минимальный графф толщины 3 с одной “столищей”

Доказательство совершенно аналогично уже разобранному случаю $m=2$ : на каждом следуюшем шаге мы просто отмечаем еще три неособые точки на компоненте $X_{0}$ и применяем леммы 5.2 и 4.1 .

Будем теперь доказывать теорему 1.2 индукцией по числу неприводимых компонент. Для трехкомпонентных кривых теорема уже доказана. Рассмотрим кривую $X$, имеюшую более трех неприводимых компонент. Предположим, что теорема уже доказана для всех кривых, имеюших меньше неприводимых компонент, чем $X$. Как и раньше, нам достаточно доказать, что ограничение канонического отображения на каждую неприводимую компоненту является вложением и что 
каноническое отображение различает пары точек, расположенных на разных неприводимых компонентах.

Рассмотрим произвольную неприводимую компоненту $X_{1}$, соответствующую вершине $v_{1}$ двойственного графа $\Gamma(X)$. Предположим, что нам удалось найти две другие вершины этого графа $v^{\prime}$ и $v^{\prime \prime}$, соединенные хотя бы одним ребром, так что индуцированный замкнутый подграф графа $\Gamma(X)$, определенный этими двумя вершинами, связен. Дополнительньй открытый подграф̆ соответствует пунктированной кривой $X^{0}=X \backslash\left(X_{v^{\prime}} \cup X_{v^{\prime \prime}}\right)$ (с отмеченными точками пересечения ее с $\left.X_{v^{\prime}} \cup X_{v^{\prime \prime}}\right)$, и ограничение канонического отображения $\varphi_{X}$ на $X^{0}$ является каноническим отображением $\varphi_{X^{0}}$ пунктированной кривой $X^{0}$ (лемма 4.1). Но каноническое отображение $\varphi_{X^{0}}$ совпадает с ограничением на нее канонического отображения ее однокомпонентной компактификации $\widehat{X}^{0}$ (лемма 4.2 ), которая уже имеет на одну неприводимую компоненту меньше, чем $X$. Толшина двойственного графа $\Gamma\left(\widehat{X}^{0}\right)$ может при этом только, возможно, возрасти, поэтому по предположению индукции каноническое отображение кривой $\widehat{X}^{0}$ является вложением и, следовательно, его ограничение на $X_{0}$ также является вложением.

Остается выяснить, всегда ли мы можем выбрать требуемые вершины $v^{\prime}$ и $v^{\prime \prime}$. Очевидно, это невозможно в единственном случае, когда все вершины соединены только с $v_{1}$ и никакие две другие вершины не соединены между собой. (Можно представлять себе $v_{1}$ как "столицу" некоторого государства, все города которого соединены дорогами только со “столицей”, но не соединены между собой.) Но в этом случае, используя лемму 5.1, мы можем удалять ребра до тех пор, пока толщина графа остается не менее трех. В конце концов мы придем к “звездному тэтаграфу", для которого теорема уже доказана.

Тем самым, мы доказали, что ограничение канонического отображения на любую неприводимую компоненту $X$ всегда является вложением.

Возьмем теперь две неособые точки на двух неприводимых компонентах $X_{1}$ и $X_{2}$, соответствующих вершинам $v_{1}$ и $v_{2}$ двойственного графа $\Gamma(X)$. Как мы уже видели, проблема различения точек, лежащих на разных компонентах, может быть сведена к случаю, когда все компоненты рациональны. Далее мы используем тот же самый прием. Предположим, что нам удалось найти две другие вершины $v^{\prime}$ и $v^{\prime \prime}$ двойственного графа $\Gamma(X)$, отличные от $v_{1}$ и $v_{2}$, соединенные хотя бы одним ребром, так что индуцированный замкнутьй подграф графа $\Gamma(X)$, определенньй этими двумя вершинами, связен. Дополнительньй открытый подграф соответствует пунктированной кривой $X^{0}=X \backslash\left(X_{v^{\prime}} \cup X_{v^{\prime \prime}}\right)$ (с отмеченными точками пересечения ее с $X_{v^{\prime}} \cup X_{v^{\prime \prime}}$ ), и ограничение канонического отображения $\varphi_{X}$ на $X^{0}$ является каноническим отображением $\varphi_{X^{0}}$ пунктированной кривой $X^{0}$ (лемма 4.1). Но каноническое отображение $\varphi_{X^{0}}$ совпадает с ограничением на $X^{0}$ канонического отображения ее однокомпонентной компактификации $\widehat{X}^{0}$ (лемма 4.2), которая уже имеет на одну неприводимую компоненту меньше, чем $X$. Толщина двойственного графа $\Gamma\left(\widehat{X}^{0}\right)$ может при этом только, возможно, возрасти, поэтому по предположению индукции каноническое отображение кривой $\widehat{X}^{0}$ является вложением. Следовательно, его ограничение на $X_{0}$ также является вложением и, тем самым, различает точки на ее компонентах $X_{1}$ и $X_{2}$.

В каком случае мы не сможем найти требуемых вершин $v^{\prime}$ и $v^{\prime \prime}$ ? Это может произойти только если все остальные вершины соединены лишш с $v_{1}$ и $v_{2}$. (Можно 
представлять себе этот случай как некоторое государство с двумя "столицами", так что все остальные города соединены дорогами только со “столицами”, но не соединены между собой.) Нетрудно проверить, что $\Gamma(X)$ в этом случае содержит один из девяти компактных подграфов толщины 3 , приведенных на рис. 7.
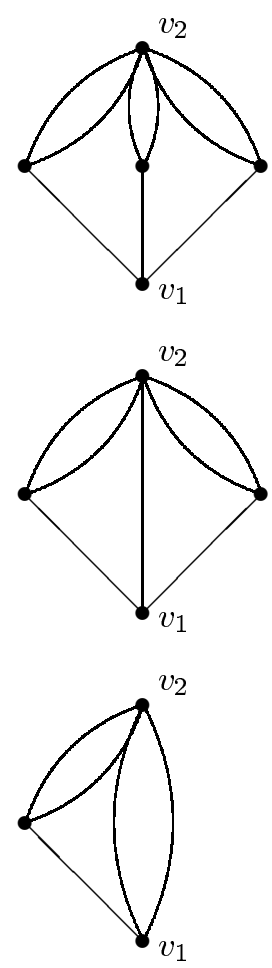
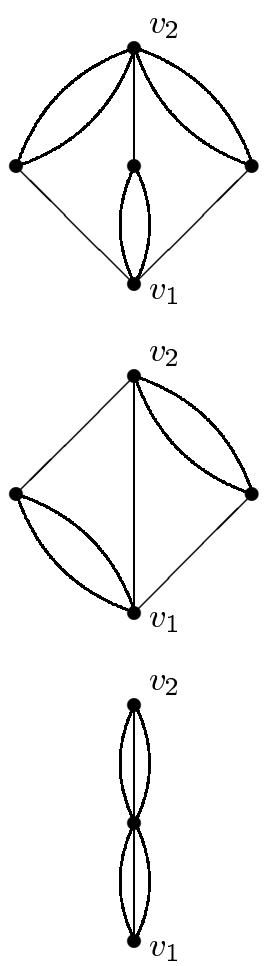
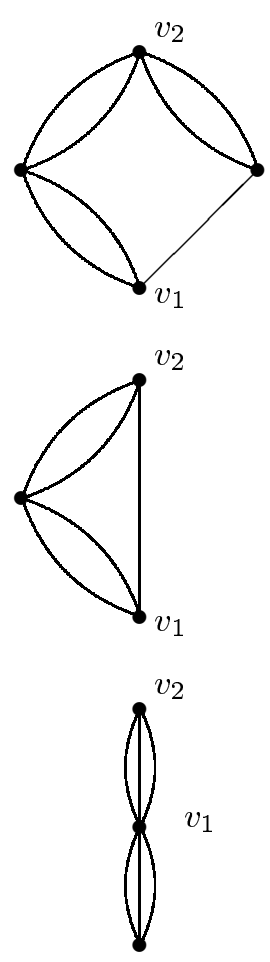

Рис. 7. Девять минимальных графов толщины 3 с двумя “столицами”

Для завершения доказательства теоремы достаточно найти на каждой из соответствующих девяти кривых регулярный дифференциал, разделяющий точки на компонентах $X_{1}$ и $X_{2}$, и продолжить его нулем на все остальные компоненты $X$. Вместо этого достаточно просто предъявить каноническое вложение каждой из девяти перечисленных кривых, что представляет собой простое упражнение. Мы опишем здесь каноническое вложение только одной, наиболее интересной кривой, соответствуюшей первому графу на рис. 7. Шестивалентная вершина соответствует кривой Веронезе степени 4 в $\mathbb{P}^{4}$, а три вершины, отличные от $v_{1}$ и $v_{2}$, соответствуют трем ее секущим прямым. Если бы какие-нибудь две секущие пересекались, они бы определяли плоскость, которая вместе с любой пятой точкой кривой порождала бы трехмерную гиперплоскость, пересекаюшую нашу кривую четвертой степени в пяти точках, что невозможно. Следовательно, любые две секущие определяют трехмерное подпространство, которое пересекает третью секущую в одной точке, и существует единственная прямая, проходящая через данную точку трехмерного пространства и пересекаюшая две заданные скрещивающиеся прямые. Эта прямая и является последней компонентой нашей кривой. 
В заключение скажем несколько слов о доказательстве теоремы 1.1, которое намного проще. Во-первых, регулярньй квадратичный дифференциал на $X$ представляет собой набор мероморфных дифференциалов на нормализациях компонент $X_{v}^{\nu}, v \in V$, имеющих полюсы не более чем второго порядка в точках $P_{\vec{e}}$ :

$$
\left.\left(\nu^{*}\left(\Omega_{X}^{1}\right)^{\otimes 2}\right)\right|_{X_{v}^{\nu}}=\left(\Omega_{X_{v}^{\nu}}^{1}\right) \otimes 2\left(\sum_{\vec{e} \in S_{-}(v)} 2 P \vec{e}\right) .
$$

Условия согласования в этом случае симметричны:

$$
\operatorname{bires}_{P_{\vec{e}}} \omega_{v_{1}}=\operatorname{bires}_{P_{\overleftarrow{e}}} \omega_{v_{2}}
$$

(определение бивычета квадратичного дифференциала см., например, в [7]).

Таким образом, получается отображение

$$
H^{0}\left(X,\left(\Omega_{X}^{1}\right)^{\otimes 2}\right) \rightarrow k^{E} .
$$

Нетрудно проверить, что для стабильной в смысле Делиня-Мамфорда кривой это отображение сюръективно. Это значит, что ограничение дважды канонического отображения кривой $X$ на каждую неприводимую компоненту $X_{v}$ является дважды каноническим отображением компоненты $X_{v}$, рассматриваемой как пунктированная кривая, соответствуюшая открытому подграфу $\Gamma(X)$, состоящему из вершины $v$ и всех исходящих из нее ребер. Дважды каноническое отображение неприводимой некомпактной пунктированной кривой не является вложением только в случаях 1) и 2) теоремы 1.1. Для стабильных компактных неприводимых кривых единственным исключением является случай арифметического рода 2: случаи $3 \mathrm{a}), 3 \mathrm{~b})$ и $3 \mathrm{c})$ теоремы 1.1 .

Проблема различения точек, расположенных на разных неприводимых компонентах кривой $X$, сводится к случаю, когда все компоненты рациональны, теми же рассуждениями, которые были использованы при доказательстве леммы 6.1. Сюръективность (6.3) позволяет тогда выбрать любые значения бивычетов в особых точках двух заданных компонент. Единственным исключением при этом будет случай $3 \mathrm{~d})$ теоремы 1.1 .

\section{Список литературы}

1. Manin Yu.I. Frobenius manifolds, quantum cohomology, and moduli spaces. Providence, RI: Amer. Math. Soc., 1999. (Amer. Math. Soc. Colloq. Publ. V. 47.)

2. Knudsen F. Projectivity of the moduli space of stable curves. II // Math. Scand. 1983. V. 52. P. 161-199.

3. Deligne P., Mumford D. The irreducibility of the space of curves of given genus // Inst. Hautes Études Sci. Publ. Math. 1969. V. 36. P. 75-109.

4. Kapranov $M$. Veronese curves and Grothendieck-Knudsen mooduli space $\bar{M}_{0, n} / /$ J. Algebraic Geom. 1993. V. 2. P. 239-262.

5. Тюрин A.H. Квантование, классическая и квантовая теория поля и тета-функция. М.-Ижевск: Ин-т компютерных исследований.

6. Гриффитс Ф., Харрис Джс. Принципы алгебраической геометрии. М.: Мир, 1982.

7. Тюрин А. Н. О периодах квадратичных дифференциалов // УМН. 1978. Т. 33. №6. C. 149-195.

Математический институт

Поступила в редакцию

им. В.А. Стеклова

24.10 .2003

E-mail: artamkin@mail.ru 